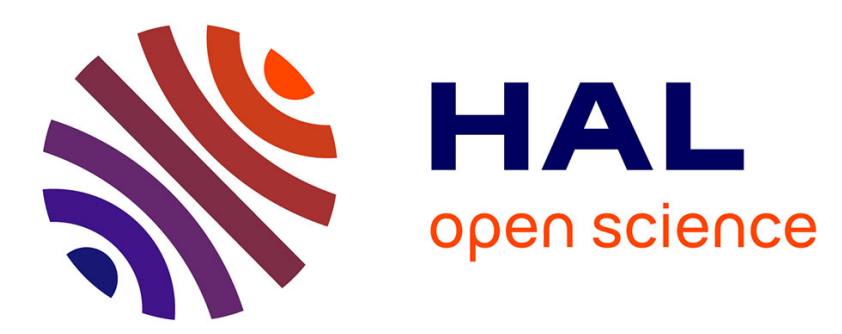

\title{
Chance-Constrained Optimization of Reliable Fixed Broadband Wireless Networks
}

\author{
Grit Classen, David Coudert, Arie Koster, Napoleao Nepomuceno
}

\section{To cite this version:}

Grit Classen, David Coudert, Arie Koster, Napoleao Nepomuceno. Chance-Constrained Optimization of Reliable Fixed Broadband Wireless Networks. INFORMS Journal on Computing, 2014, 26 (4), pp.893-909. 10.1287/ijoc.2014.0605 . hal-01070780

\author{
HAL Id: hal-01070780 \\ https://hal.inria.fr/hal-01070780
}

Submitted on 10 Feb 2015

HAL is a multi-disciplinary open access archive for the deposit and dissemination of scientific research documents, whether they are published or not. The documents may come from teaching and research institutions in France or abroad, or from public or private research centers.
L'archive ouverte pluridisciplinaire HAL, est destinée au dépôt et à la diffusion de documents scientifiques de niveau recherche, publiés ou non, émanant des établissements d'enseignement et de recherche français ou étrangers, des laboratoires publics ou privés. 


\title{
Chance-Constrained Optimization of Reliable Fixed Broadband Wireless Networks
}

\author{
Grit Claßen*1, David Coudert ${ }^{2,3}$, Arie M. C. A. Koster ${ }^{1}$ and Napoleão Nepomuceno ${ }^{4}$ \\ ${ }^{1}$ RWTH Aachen University, Lehrstuhl II für Mathematik, Aachen, Germany \\ ${ }^{2}$ Inria, France \\ ${ }^{3}$ Univ. Nice Sophia Antipolis, CNRS, I3S, UMR 7271, 06900 Sophia Antipolis, France \\ ${ }^{4}$ Universidade de Fortaleza, Programa de Pós-Graduação em Informática Aplicada, \\ Fortaleza, Brazil
}

\begin{abstract}
In this paper, we extend our former investigation on conceiving reliable fixed point-topoint wireless networks under outage probability constraints CCKN11b; CCKN11a. We consider the problem of determining the minimum cost bandwidth assignment of a network, while guaranteeing a reliability level of the solution. If the optimal bandwidth assignment and routing of traffic demands are accomplished, the reliability criterion requires that network flows remain feasible with high probability, regarding that the performance of microwave links is prone to variations due to external factors, e.g., weather. We introduce a chance-constrained programming approach to tackle this problem and we present reformulations to standard Integer Linear Programming (ILP) models, including a budget constrained formulation. To improve the solving performance, we propose new valid inequalities and a primal heuristic. Computational results present a performance analysis of the valid inequalities and the heuristic. Further, the outperformance of the novel model compared to more traditional approaches is documented.
\end{abstract}

Keywords: fixed wireless networks; capacitated network design; network reliability; chance-constrained programming; integer programming.

\section{Introduction}

Fixed point-to-point wireless communications is a particular sector of the communication industry that holds great promise for delivering private high-speed data connections by means of microwave radio transmission [And03]. Microwave, in the context of this work, refers to terrestrial fixed point-to-point digital radio communications, usually employing highly directional antennas in clear line-of-sight and operating in licensed frequency bands from $6 \mathrm{GHz}$ to $38 \mathrm{GHz}$. This makes microwave communications typically free of interference. The antennas used to transmit and receive the signal into/from free space are usually located at the top of communication towers. Two radios are required to establish a microwave link, whose capacity can attain 500 Mbps nowadays, between two locations that can be several kilometers apart, up to $50 \mathrm{~km}$.

Historically, microwave was mainly used by incumbent network operators to carry trunk telephony traffic, and by broadcasters to link remote broadcast transmitters to studios. Today,

\footnotetext{
${ }^{*}$ Corresponding author, classen@math2.rwth-aachen.de
} 
demand is driven by the infrastructure requirements of mobile networks, where microwave is used to provide interconnectivity between base stations, controllers, and switches [BKC01]. In fact, thanks to the ability for microwave links to be rapidly and cost-effectively deployed, fixed point-to-point wireless networks have become a common alternative to provide broadband communications, particularly in emerging countries and remote locations where classical copper or fiber lines are too costly or simply unavailable to cope with the increasing demand for bandwidth-intensive services [Leh10]. It is not a coincidence that over $50 \%$ of the world's base transceiver stations are connected using microwave technologies [Lit09].

Despite recent advances in fixed point-to-point wireless communications, a variety of questions remain unaddressed in this area. Particularly, capacity planning in wireless networks is quite different from wired network planning. In fact, the radio frequency spectrum is a limited natural resource which has been regulated worldwide to promote its efficient use. Moreover, environment conditions, such as weather, play an important role in wireless communications since they can introduce instantaneous variations into the communication channel, likely leading to outage events.

Although having limited bandwidth and suffering channel impairments, fixed point-to-point wireless networks must degrade smoothly as environment conditions degrade. As a common practice, operators highly overprovision bandwidth during network planning to avoid traffic bottlenecks under adverse scenarios such as the performance of some deteriorated links. This approach, however, incurs additional investments that do not result in resource- and cost-efficient networks, besides leading to an inefficient use of the radio spectrum. Therefore, establishing better wireless networks is not just a matter of adding bandwidth but it also entails a complex design decision aiming at enhancing network's reliability to cope with channel fluctuations.

The most common idea of network reliability in the literature is a numerical parameter which represents the probability that a subset of nodes in a probabilistic network is connected. Computing the network reliability is known to be a computationally difficult problem [Bal80; Bal86], even for the case in which the subset of nodes is restricted to a single source-destination pair, viz. the two-terminal network reliability problem [PB84; BJ88]. To the best of our knowledge, [DBHRX07] is the only work to investigate the reliability of fixed broadband wireless networks under outage probability events. The authors, however, do not consider traffic requirements and they assume that the network is uncapacitated. In addition, the variation on the performance of a microwave link is not taken into account, but only unqualified failures that cause the complete disruption of the communication channel. Assuming that links fail independently, the authors apply currently available algorithms for the two-terminal network reliability problem and present results for a network with 5 nodes and 7 links. In And03, correlated rain fades are studied but could not be applied due to the lack of statistical information on rain cells.

The state of the art performed in the industry is to consider individual links. Ideally, the assignment of bandwidths in a network under outage probability constraints should be modeled using dependent random variables. As a first step beyond the state of the art, we consider a network without correlation between different links in this paper. Further, we propose a framework in Section 3.5 to deal with dependent random variables in which the results we derive in case of independent random variables serve as a building block.

First, we introduce a chance-constrained mathematical programming approach to conceive reliable fixed point-to-point wireless networks under outage probability constraints. Chanceconstrained programming is a specific model of stochastic optimization for dealing with random parameters in optimization problems Pré95; SDR09. Actually, there exist situations in which constraint violation can hardly be avoided because of unexpected extreme events. This approach thus aims at determining optimal decisions that have to be made prior to the observation of random parameters and remain feasible for a given infeasibility probability tolerance. Chance- 
constrained programming is still considered as hard and widely intractable since the feasible region defined by a probabilistic constraint is generally not convex. In addition, among the vast literature on chance-constrained programming, few research work has been carried out to tackle combinatorial problems [Klo10; LAN10; BB10].

Given these difficulties, we derive equivalent ILP formulations for this problem. After introducing a Big- $M$ ILP formulation for the general case, which is computationally intractable for practical instances of this problem, we concentrate on the case where the outages of microwave links are independent. We prove the equivalence of the Big-M ILP formulation and an ILP formulation in case of independent link outages. Our computational study is performed for a budget constrained formulation. Furthermore, we introduce valid inequalities and a primal heuristic to improve the solving performance for the presented model. We use typical values for the radio parameters as frequency band, bandwidth, modulation schemes, etc., and employ a largely accepted fading model, viz., Vigants-Barnett model [Bar72, Vig75], to generate realistic test instances. Our computational results evaluate the performance of the valid inequalities as well as the primal heuristic. Finally, we present a reliability analysis of fixed point-to-point wireless networks based on different budgets.

The remainder of this paper is organized as follows. In Section 2, we discuss some relevant considerations on spectrum pricing in licensed frequency bands and convey more information with regard to the link characterization, focusing on channel capacity and link availability. In Section 3, we introduce a chance-constrained formulation and its ILP counterparts for the application considered here. Furthermore, a budget constrained formulation is presented. Section 4 is devoted to cutset-based valid inequalities to improve the dual bounds of the ILP formulation and a primal heuristic to improve the primal bounds. In Section 5, we evaluate the performance of the proposed cutset inequalities and the heuristic and present a reliability analysis for various budgets in different network topologies. Some final remarks and comments on future work conclude the paper with Section 6 .

\section{Preliminaries}

\subsection{Spectrum Pricing}

The radio frequency spectrum is a limited natural resource regulated worldwide by the International Telecommunications Union (ITU) [ITU12. In conjunction with ITU regulations, national legislation instruments establish the availability of frequency bands for specific applications and the procedures for issuing licenses, as well as the rights and obligations resulting from using the spectrum. A license (assignment) is the authorization given by an administration for a radio station to use a radio frequency under specified conditions. Obtaining a license requires a careful review and functional understanding of the administrative rules that govern the use of the frequency spectrum, and it normally incurs the payment of charges.

The value of the spectrum largely depends on its physical properties, notably the available bandwidth, geographic range, and reuse capability, that determine its ability to convey information under a wide variety of scenarios [BKC01]. In contrast with terrestrial broadcasting and wide area mobile communications which require lower frequency bands to provide wide area non line-of-sight coverage and are normally awarded through auctions, terrestrial fixed point-to-point links or satellite systems, which can take advantage of the bandwidth available in higher frequency bands, are usually licensed through registration in national database on a first-in-time is first-in-right basis. Besides, fixed point-to-point services often require careful frequency assignment and coordination, which makes auctions potentially unwieldy.

Nowadays, growing demand for microwave links for applications such as mobile network 
infrastructure has led to increasing pressure on the available spectrum, prompting the introduction of administrative pricing in an attempt to promote the economical and efficient use of spectrum. In this context, charges can take the form of simply setting fees sufficient to recover the costs of spectrum management or can be used to guide users in making decisions to ensure the optimal use of scarce resources. In addition, administrative pricing like fixing lower charges to frequency bands and/or locations that are not congested may also be applied where there is no scarcity.

Because of specific differences from country to country, a comprehensive tabulation of spectrum prices is beyond the scope of this paper. The interested reader is referred to an extensive study on pricing of frequency spectrum in BKC01 for more details. As it is typically done, we assume that the price of a frequency spectrum for a single microwave link is a function of the amount of spectrum (bandwidth) in $\mathrm{MHz}$ with which a license is associated.

\subsection{Link Characterization}

Commonly, to support broadband applications, modern microwave systems use quadrature amplitude modulation (QAM). An $m$-QAM scheme presents $m$ combinations of amplitude and phase, each one representing an $n$-bit pattern called a symbol, with $n=\log _{2} m$ and integer. Given the channel bandwidth $W$ and the $m$-QAM scheme in use, we can approximate the channel capacity $C$ by

$$
C[b p s]=n \cdot W[H z] .
$$

High-level QAM schemes, despite presenting better bandwidth efficiency, are more susceptible to errors due to channel impairments. As the modulation scheme changes to accommodate higher data rates, the signal-to-noise ratio (SNR) requirement increases to preserve the bit error rate (BER) (see Table 1). Rigorously, we can also use different error correction codes. In any case, we can rebuild Table 1 for different combinations of modulation and coding (and other radio parameters) based on equipment specifications.

Table 1: Bandwidth efficiency, SNR requirement, and capacity.

\begin{tabular}{lccccc}
\hline $\begin{array}{l}\text { Modulation } \\
\text { scheme }\end{array}$ & $\begin{array}{c}\text { Bandwidth efficiency } \\
\text { (bps/Hz) }\end{array}$ & $\begin{array}{c}\text { SNR requirem. } \\
(\mathrm{dB})\end{array}$ & $\begin{array}{c}\text { Capacity f. 7 MHz } \\
\text { (Mbps) }\end{array}$ & $\begin{array}{c}\text { Capacity f. 14 MHz } \\
\text { (Mbps) }\end{array}$ & $\begin{array}{c}\text { Capacity f. 28 MHz } \\
\text { (Mbps) }\end{array}$ \\
\hline QPSK & 2 & 14.21 & 14 & 28 & 56 \\
16-QAM & 4 & 21.02 & 28 & 56 & 112 \\
32-QAM & 5 & 25.24 & 35 & 70 & 140 \\
64-QAM & 6 & 27.45 & 42 & 84 & 168 \\
128-QAM & 7 & 31.10 & 49 & 98 & 196 \\
256-QAM & 8 & 33.78 & 56 & 112 & 224 \\
\hline
\end{tabular}

Since the transmitted signal suffers deep fades, microwave links are susceptible to outage events. Fading phenomena are described in statistical terms and the probability of fades of a particular magnitude can be evaluated through analytical techniques Bar72; Vig75; Cra96. To overcome outage events, modern microwave systems employ adaptive modulation and coding which has been proven to considerably enhance link performance GC97; GC98]. To keep the BER performance, this technique entails the variability of the link's capacity.

Note that, on the one hand, the assigned bandwidth for each microwave link is a network engineer's decision subject to obtaining licenses upon payment of renewal fees. On the other hand, in response to channel fluctuations, the radio configuration is a random factor. Considering a finite set of efficient radio configurations [CNR10], for which no configuration that presents better bandwidth efficiency for a lower SNR requirement exists, we can associate a 
discrete probability distribution with these configurations. We obtain the probability distribution either from statistical studies (in case of license renewal of a network in operation) or from fading models and power budget calculations. We henceforth assume that such a discrete probability distribution is known for each microwave link and bandwidth.

\section{Mathematical Formulations}

In this section, we introduce a chance-constrained mathematical formulation and its ILP counterparts for the optimization problem of deciding both the bandwidth assignment and the network flows that minimize the total bandwidth cost while handling all the traffic requirements simultaneously with a given reliability level. Furthermore, we reformulate this model to maximize the network reliability while a budget constraint is fulfilled.

\subsection{Chance-Constrained Formulation}

The described problem can be formally stated as follows. The network's topology is modeled as a digraph $G=(\mathcal{V}, \mathcal{A})$, where each node $v \in \mathcal{V}$ denotes a radio base station (RBS) and each arc $u v \in \mathcal{A}$ represents a microwave link from $u$ to $v$, with $u, v \in \mathcal{V}$ and $u \neq v$. Let $\delta^{+}(v)$ $\left(\delta^{-}(v)\right)$ denote the set of outneighbors (inneighbors) of $v$. Let $P_{u v}$ be the number of bandwidth choices available for arc $u v \in \mathcal{A}$. Each bandwidth $b_{u v}^{p}, p=1, \ldots, P_{u v}$, is associated with its $\operatorname{cost} c_{u v}^{p}$ and a random variable $\eta_{u v}^{p}$ that represents the bandwidth efficiency of the current radio configuration which varies over time in response to channel fluctuations. Let $\varepsilon>0$ be the infeasibility tolerance (typically near zero) chosen by the network engineer. The traffic requirements are modeled by a set $\mathcal{K}$. For each $k \in \mathcal{K}, s^{k}$ denotes the origin, $t^{k}$ the destination, and $d^{k}>0$ the expected demand.

We aim at determining the bandwidth assignment and the traffic flows that minimize the total bandwidth cost. Let $y_{u v}^{p}$ be the binary decision variable indicating whether bandwidth $b_{u v}^{p}$, $p=1, \ldots, P_{u v}$, is assigned or not to arc $u v \in \mathcal{A}$. The flow variables $x_{u v}^{k}$ denote the amount of $d^{k}, k \in \mathcal{K}$, routed on arc $u v \in \mathcal{A}$. The optimization problem can be formulated as follows.

$$
\begin{aligned}
& \min \sum_{u v \in \mathcal{A}} \sum_{p=1}^{P_{u v}} c_{u v}^{p} y_{u v}^{p} \\
& \text { s.t. } \sum_{u \in \delta^{-}(v)} x_{u v}^{k}-\sum_{u \in \delta^{+}(v)} x_{v u}^{k}=\left\{\begin{array}{ll}
-d^{k}, & \text { if } v=s^{k}, \\
d^{k}, & \text { if } v=t^{k}, \\
0, & \text { otherwise }
\end{array} \quad \forall v \in \mathcal{V}, \quad \forall k \in \mathcal{K}\right. \\
& \mathbb{P}\left[\sum_{k \in \mathcal{K}} x_{u v}^{k} \leq \sum_{p=1}^{P_{u v}} \eta_{u v}^{p} b_{u v}^{p} y_{u v}^{p} \quad \forall u v \in \mathcal{A}\right] \geq 1-\varepsilon \\
& \sum_{p=1}^{P_{u v}} y_{u v}^{p} \leq 1 \\
& x_{u v}^{k} \geq 0, y_{u v}^{p} \in\{0,1\} \\
& \forall u v \in \mathcal{A}, \forall k \in \mathcal{K}, p=1, \ldots, P_{u v}
\end{aligned}
$$

The objective function (1a) represents the total bandwidth cost that is to minimize. The flow conservation property is expressed by $(1 \mathrm{~b})$. It provides the routes for each demand pair, guaranteeing that the traffic requirements are entirely fulfilled. Constraint (1c) enforces an infeasibility tolerance on the entire block of capacity constraints, guaranteeing that the assigned bandwidth supports all the traffic to be routed through the network with (high) probability 
$1-\varepsilon$. Finally, the bandwidth assignment is determined by $(1 \mathrm{~d})$. For each arc, it allows a single selection among the available bandwidth choices.

\section{$3.2 \quad B i g-M$ ILP Formulation}

A first way to reformulate the chance-constrained model (1) as an integer linear program (cf. LAN10; Rus02]) requires the application of big- $M$ type constraints, where $M$ is a sufficiently large constant. For this purpose, we consider a finite number of realizations $\eta^{1}, \ldots, \eta^{R}$ of the random vector $\eta$ consisting of the random variables $\eta_{u v}^{p}$. The realizations occur with probability $\pi_{1}, \ldots, \pi_{R}$ (with $\sum_{r=1}^{R} \pi_{r}=1$ ). Let $z_{r}, r=1, \ldots, R$ be binary variables, where $z_{r}=0$ guarantees that the capacity constraints are satisfied taking into account realization $\eta^{r}$. We set $M:=\sum_{k \in \mathcal{K}} d^{k}$. Then (1) can be rewritten as

$$
\begin{aligned}
& \min (1 \mathrm{a}) \\
& \text { s.t. } 1 \mathrm{1 \textrm {b }}, \mathrm{1 \textrm {d }}) \\
& \sum_{k \in \mathcal{K}} x_{u v}^{k}-M z_{r} \leq \sum_{p=1}^{P_{u v}}\left(\eta^{r}\right)_{u v}^{p} b_{u v}^{p} y_{u v}^{p} \quad \forall u v \in \mathcal{A}, r=1, \ldots, R \\
& \sum_{r=1}^{R} \pi_{r} z_{r} \leq \varepsilon \\
& \begin{array}{l}
x_{u v}^{k} \geq 0, y_{u v}^{p} \in\{0,1\} \\
z_{r} \in\{0,1\} \quad \forall u v \in \mathcal{A}, \forall k \in \mathcal{K}, p=1, \ldots, P_{u v} \\
\forall r=1, \ldots, R .
\end{array}
\end{aligned}
$$

The knapsack constraint $2 \mathrm{dd}$ ) is equivalent to the probabilistic constraint

$$
\sum_{r=1}^{R} \pi_{r}\left(1-z_{r}\right) \geq 1-\varepsilon .
$$

The big- $M$ constraints (2c) in association with the knapsack inequality (2d) guarantee that the probability of scenarios which do not satisfy the capacity constraints is less than or equal to the infeasibility tolerance $\varepsilon$, thus enforcing the probabilistic constraint (1c).

In general, the (merely unknown) correlation among outage events of different radio links prohibits the computation of the probabilities $\pi_{r}$. Under the assumption that microwave links suffer fades independently (but $\eta_{u v}^{p} \forall p=1, \ldots, P_{u v}$ on a single link $u v \in \mathcal{A}$ are not assumed to be independent), we can define an artificial set of realizations. Due to $(1 \mathrm{~d})$, at most one bandwidth per link is selected. Therefore, the dependency between the bandwidth efficiencies $\eta_{u v}^{p}, p=$ $1, \ldots, P_{u v}$ does not play a role in the probability calculation as shown in the following.

By the independence between the links, we can limit the discussion to a single link $u v \in \mathcal{A}$. Let $Q^{p}$ be the number of bandwidth efficiencies for the chosen link $u v$ and bandwidth choice $p$. Further, let $\mathcal{D}^{p}$ be the domain, i.e., the possible bandwidth efficiencies, of the random variable $\eta_{u v}^{p}$ and define a bijection $f^{p}: \mathcal{D}^{p} \rightarrow\left\{1, \ldots, Q^{p}\right\}$ with $f^{p}\left(\eta_{u v}^{p}\right)=q$ mapping bandwidth efficiency to radio configuration. For a fixed bandwidth choice $\tilde{p}$, the probability that $u v$ runs with radio configuration $\nabla \in\left\{1, \ldots, Q^{\tilde{p}}\right\}$ is $\mathbb{P}\left[f\left(\eta_{u v}^{\tilde{p}}\right)=\nabla\right]=\sum_{r=1 \mid f\left(\left(\eta^{r}\right)_{u v}^{\tilde{p}}\right)=\nabla}^{R} \pi_{r}$. Now, we define all possible bandwidth-independent realizations with probabilities $\pi_{r}^{*}$ such that the probability $\mathbb{P}\left[f\left(\eta_{u v}^{\tilde{p}}\right)=\nabla\right]$ can be determined in the same way as before. More precisely, let $R^{*}=\prod_{p=1}^{P_{u v}} Q^{p}$ and $\pi_{r}^{*}:=\prod_{p=1}^{P_{u v}} \mathbb{P}\left[\eta_{u v}^{p}=\left(\eta^{r}\right)_{u v}^{p}\right]$ for $r=1, \ldots, R^{*}$. 
Lemma 1. Let $\tilde{p} \in\left\{1, \ldots, P_{u v}\right\}$ be a bandwidth choice and $\nabla \in\left\{1, \ldots, Q^{\tilde{p}}\right\}$ a radio configuration. It holds that

$$
\sum_{r=1 \mid f\left(\left(\eta^{r}\right)_{u v}^{\tilde{p}}\right)=\nabla}^{R^{*}} \pi_{r}^{*}=\mathbb{P}\left[f\left(\eta_{u v}^{\tilde{p}}\right)=\nabla\right] .
$$

Proof. By definition of $\pi_{r}^{*}$, we obtain

$$
\begin{aligned}
\sum_{r=1 \mid f\left(\left(\eta^{r}\right)_{u v}^{\tilde{p}}\right)=\nabla}^{R^{*}} \pi_{r}^{*} & =\sum_{r=1 \mid f\left(\left(\eta^{r}\right)_{u v}^{\tilde{p}}\right)=\nabla}^{R^{*}}\left(\prod_{p=1}^{P_{u v}} \mathbb{P}\left[\eta_{u v}^{p}=\left(\eta^{r}\right)_{u v}^{p}\right]\right) \\
& =\mathbb{P}\left[f\left(\eta_{u v}^{\tilde{p}}\right)=\nabla\right] \cdot \underbrace{\sum_{r=1 \mid f\left(\left(\eta^{r}\right)_{u v}^{\tilde{p}}\right)=\nabla}^{R^{*}}\left(\prod_{p=1 \mid p \neq \tilde{p}}^{P_{u v}} \mathbb{P}\left[\eta_{u v}^{p}=\left(\eta^{r}\right)_{u v}^{p}\right]\right)}_{(\star)}
\end{aligned}
$$

It remains to show that $(\star)=1$. For this purpose, we fix another bandwidth choice $\bar{p} \in$ $\left\{1, \ldots, P_{u v}\right\} \backslash\{\tilde{p}\}$ and separate all corresponding summands (regarding the radio configuration) as follows.

$$
\begin{aligned}
(\star) & =\sum_{q=1}^{Q^{\bar{p}}}\left[\mathbb{P}\left[f\left(\eta_{u v}^{\bar{p}}\right)=q\right] \cdot \sum_{r=1 \mid f\left(\left(\eta^{r}\right)_{u v}^{\tilde{p}}\right)=\nabla}^{R^{*}}\left(\prod_{p=1 \mid p \neq \tilde{p}, \bar{p}}^{P_{u v}} \mathbb{P}\left[\eta_{u v}^{p}=\left(\eta^{r}\right)_{u v}^{p}\right]\right)\right] \\
& =\sum_{r=1 \mid f\left(\left(\eta^{r}\right)_{u v}^{\tilde{p}}\right)=\nabla}^{R^{*}}\left(\prod_{p=1 \mid p \neq \tilde{p}, \bar{p}}^{P_{u v}} \mathbb{P}\left[\eta_{u v}^{p}=\left(\eta^{r}\right)_{u v}^{p}\right]\right) \cdot \underbrace{\sum_{q=1}^{Q^{\bar{p}}} \mathbb{P}\left[f\left(\eta_{u v}^{\bar{p}}\right)=q\right]}_{=1}
\end{aligned}
$$

Separating all bandwidth choices subsequently that way, it follows $(\star)=1$ and the proof is complete.

Nevertheless, this model is highly intractable due to the very large number of scenarios to be considered. In addition, big- $M$ models are often numerically unstable. In the sequel, we propose a computationally more tractable ILP model in case of independent link outages.

\subsection{ILP Formulation in Case of Independent Link Outages}

If the link outages are independent, we can reformulate the left hand side of $(1 \mathrm{c})$ as the product of probabilities. For this, we introduce the following notation. Let $Q_{u v}^{p}$ be the number of configurations possible for arc $u v$ with respect to the bandwidth choice $p$. Let $\rho_{u v}^{p q}$ be the probability that arc $u v$, assuming bandwidth choice $p$, is running at configuration $q$ or higher, i.e., the $m$ in an $m$-QAM modulation scheme is higher. Remember that higher configurations are more bandwidth-efficient (cf. Table 1), but less robust in the sense that they are more susceptible to channel impairments. Now $b_{u v}^{p q}$ represents the capacity on arc $u v$ for a given bandwidth choice $p$ and a specific configuration $q$. In addition, the binary decision variables $y$ obtain a new index $q$ that incorporates the assumption on the configuration. The assumption on the network configuration actually encompasses as well all the network configurations that are more bandwidth-efficient, i.e, a feasible routing of traffic demands to an arc uv operating at bandwidth choice $p$ and running at configuration $q$ is also feasible if the arc runs at configurations higher than $q$. Finally, to avoid a zero-product while rewriting constraint (1c) as the product of 
probabilities, let us associate slack variables $y_{u v}^{0}, u v \in \mathcal{A}$, with constraints $\left(1 \mathrm{~d}\right.$ ), where $y_{u v}^{0}=1$ indicates that arc $u v$ is not operated. In this case, the capacity of the arc is known to be 0 with probability of 1 . The problem can then be written as follows.

$$
\begin{array}{ll}
\min & \sum_{u v \in \mathcal{A}} \sum_{p=1}^{P_{u v}} \sum_{q=1}^{Q_{u v}^{p}} c_{u v}^{p} y_{u v}^{p q} \\
\text { s.t. } & \sum_{u \in \delta^{-}(v)} x_{u v}^{k}-\sum_{u \in \delta^{+}(v)} x_{v u}^{k}= \begin{cases}-d^{k}, & \text { if } v=s^{k} \\
d^{k}, & \text { if } v=t^{k} \\
0, & \text { otherwise }\end{cases}
\end{array} \quad \forall v \in \mathcal{V}, \forall k \in \mathcal{K}
$$

In the capacity constraints (3c), we assume explicitly a hypothesis on the radio configuration. For a given arc and bandwidth, the lower the configuration is, the lower the bandwidth efficiency assumed to this arc in time of design will be and also the higher the probability that the effective capacity on this arc in time of operation supports all the traffic to be routed through it will be. In other words, more conservative hypotheses on the radio configuration lead to more reliable solutions. Constraint (3d) denotes formally this relation. According to the bandwidth assignment and the hypotheses on the radio configuration, it guarantees that the confidence of the solutions is at least $1-\varepsilon$.

Theorem 2. Formulations (2) and (3) are equivalent in case of independent link outages.

Proof. As before, let $\mathcal{D}_{u v}^{p}$ be the domain of the random variable $\eta_{u v}^{p}$ and use the bijection $f_{u v}^{p}: \mathcal{D}_{u v}^{p} \rightarrow\left\{1, \ldots, Q_{u v}^{p}\right\}$ with $f_{u v}^{p}\left(\eta_{u v}^{p}\right)=q$ from Section 3.2 which maps bandwidth efficiency to radio configuration. For the sake of simplicity, we write $f$ instead of $f_{u v}^{p}$ here.

Now, we prove that, for every feasible bandwidth assignment and routing of traffic demands to formulation (2), there exists a corresponding feasible solution to formulation (3) with same cost, and vice versa. Given a feasible solution $(\tilde{x}, \tilde{y}, \tilde{z})$ to formulation (2), assume without loss of generality that it includes all feasible realizations, i.e., for $r=1, \ldots, R$,

$$
\tilde{z}_{r}=0 \Longleftrightarrow \sum_{k \in \mathcal{K}} \tilde{x}_{u v}^{k} \leq \sum_{p=1}^{P_{u v}}\left(\eta^{r}\right)_{u v}^{p} b_{u v}^{p} \tilde{y}_{u v}^{p} \quad \forall u v \in \mathcal{A} .
$$

One can easily obtain a feasible solution $(\tilde{x}, \bar{y})$ to $(3)$ with the same cost. Since the routing of traffic demands is static for all realizations, for each arc uv, capacity constraints (2c) must be satisfied for all feasible realizations. Let us define $\mathcal{A}_{1}=\left\{u v \in \mathcal{A} \mid \sum_{p=1}^{P_{u v}} \tilde{y}_{u v}^{p}=1\right\}$ and $\mathcal{A}_{0}=$ $\mathcal{A} \backslash \mathcal{A}_{1}$ as the sets of installed and non-installed arcs, respectively, and let $\tilde{p}_{u v}, u v \in A_{1}$, be the bandwidth choice for arc $u v$, i.e., $\tilde{y}_{u v}^{\tilde{p}}=1$. (For simplicity, whenever it is understood from 
the context, we write $\tilde{p}$ instead of $\left.\tilde{p}_{u v}.\right)$. We now define $\tilde{q}_{u v}:=f\left(\min _{r=1, \ldots, R}\left\{\left(\eta^{r}\right)_{u v}^{\tilde{p}} \mid \tilde{z}_{r}=0\right\}\right)$, $\forall u v \in \mathcal{A}_{1}$ and write $\tilde{q}$ instead of $\tilde{q}_{u v}$ whenever it is unambiguous. Note, $\tilde{z}_{r}=0 \forall r=1, \ldots, R$ with $\left(\eta^{r}\right)_{u v}^{\tilde{p}} \geq f^{-1}\left(\tilde{q}_{u v}\right)$ at all arcs $u v$ simultaneously. Then we set $\bar{y}_{u v}^{\tilde{p} \tilde{q}}:=1$ for all $u v \in \mathcal{A}_{1}$ and 0 otherwise. Besides, we set $\bar{y}_{u v}^{0}:=1, \forall u v \in \mathcal{A}_{0}$, and $\bar{y}_{u v}^{0}:=0, \forall u v \in \mathcal{A}_{1}$. Note that, from (2a) and (3a), both solutions present the same bandwidth cost and constraints (3b), (3c), and (3e) are fulfilled with $b_{u v}^{p q}=f^{-1}(q) \cdot b_{u v}^{p}$. For feasibility, we have to prove that the reliability constraint (3d) is fulfilled. For this purpose, we introduce the following notation. Taking into account the bandwidth assignment $\tilde{y}$, we reduce the space of the random vector $\eta$ to consider only the variables $\eta_{u v}^{\tilde{p}}, \forall u v \in \mathcal{A}_{1}$. Let $\tilde{\eta}$ be this reduced random vector. Again we have to deal with a finite number of realizations $\tilde{\eta}^{1}, \ldots, \tilde{\eta}^{\tilde{S}}$ of the random vector $\tilde{\eta}$. Consider the set $\tilde{\mathcal{S}}=\left\{\tilde{\eta}^{s} \mid s=1, \ldots, \tilde{S}\right.$ and $\left.f\left(\left(\tilde{\eta}^{s}\right)_{u v}^{\tilde{p}}\right) \geq \tilde{q}_{u v} \forall u v \in \mathcal{A}_{1}\right\}$ of feasible realizations of $\tilde{\eta}$ with respect to solution $\tilde{y}$. Then we have

$$
\begin{aligned}
& \prod_{u v \in \mathcal{A}}\left(\bar{y}_{u v}^{0}+\sum_{p=1}^{P_{u v}} \sum_{q=1}^{Q_{u v}^{p}} \rho_{u v}^{p q} \bar{y}_{u v}^{p q}\right)=\prod_{u v \in \mathcal{A}_{0}}(\underbrace{\bar{y}_{u v}^{0}}_{=1}+\sum_{p=1}^{P_{u v}} \sum_{q=1}^{Q_{u v}^{p}} \rho_{u v}^{p q} \underbrace{\bar{y}_{u v}^{p q}}_{=0}) \cdot \prod_{u v \in \mathcal{A}_{1}}(\underbrace{\bar{y}_{u v}^{0}}_{=0}+\sum_{p=1}^{P_{u v}} \sum_{q=1}^{Q_{u v}^{p}} \rho_{u v}^{p q} \bar{y}_{u v}^{p q}) \\
= & \prod_{u v \in \mathcal{A}_{1} p=1} \sum_{q u}^{P_{u v}} \sum_{q u}^{Q_{u v}^{p}} \rho_{u v}^{p q} \bar{y}_{u v}^{p q}=\prod_{u v \in \mathcal{A}_{1}}(\rho_{u v}^{\tilde{p} \tilde{q}} \underbrace{\bar{y}_{u v}^{\tilde{p} \tilde{q}}}_{=1})=\prod_{u v \in \mathcal{A}_{1}} \mathbb{P}\left[f\left(\eta_{u v}^{\tilde{p}}\right) \geq \tilde{q}_{u v}\right]=\sum_{s=1 \mid \tilde{\eta}^{s} \in \tilde{\mathcal{S}}} \prod_{u v \in \mathcal{A}_{1}} \mathbb{P}\left[\eta_{u v}^{\tilde{p}}=\left(\tilde{\eta}^{s}\right)_{u v}^{\tilde{p}}\right] \\
\stackrel{(+)}{=} & \sum_{r=1}^{R} \prod_{\tilde{z}_{r}=0} \prod_{u v \in \mathcal{A}}^{P_{u v}} \mathbb{P}\left[\eta_{u v}^{p}=\left(\eta^{r}\right)_{u v}^{p}\right]=\sum_{r=1 \mid \tilde{z}_{r}=0}^{R} \pi_{r}=\sum_{r=1}^{R} \pi_{r}\left(1-z_{r}\right) \geq 1-\varepsilon .
\end{aligned}
$$

For $(+)$, we use a similar argumentation as in the proof of Lemma 1 . Hence, $(\tilde{x}, \bar{y})$ is a feasible solution for (3).

Conversely, given a feasible solution $(\tilde{x}, \bar{y})$ to formulation (3), one can obtain a feasible solution $(\tilde{x}, \tilde{y}, \tilde{z})$ to $(2)$ with the same cost. We set $\tilde{y}_{u v}^{p}:=\sum_{q=1}^{Q_{u v}^{p}} \bar{y}_{u v}^{p q}, u v \in \mathcal{A}, p=1, \ldots, P_{u v}$ and define $\bar{p}_{u v}, \bar{q}_{u v}$ such that $\bar{y}_{u v}^{\bar{p}_{u v} \bar{q}_{u v}}=1$ for all $u v \in \mathcal{A}_{1}$, where $\mathcal{A}_{1}$ is defined as before. Again we write $\bar{p}$ and $\bar{q}$ for simplicity. For $r=1, \ldots, R$, we set

$$
\tilde{z}_{r}:=0 \Longleftrightarrow f\left(\left(\eta^{r}\right)_{u v}^{\bar{p}}\right) \geq \bar{q}_{u v} \forall u v \in \mathcal{A}_{1} .
$$

Again, from (2a) and (3a), both solutions present the same bandwidth cost and constraints (1b), (1d), and (2c) are fulfilled. To show that constraint (2d) is fulfilled, we follow the same argumentation as before in (*), just in the reverse direction and by replacing $\tilde{p}$ and $\tilde{q}$ by $\bar{p}$ and $\bar{q}$.

Therefore, formulations (2) and (3) are equivalent.

Note that constraint (3d) is not linear, but it can easily be linearized as follows. By employing monotonicity of logarithmic functions and because the logarithm of a product is equal to the sum of the logarithms, (3d) is equivalent to

$$
\sum_{u v \in \mathcal{A}} \log \left(1 \cdot y_{u v}^{0}+\sum_{p=1}^{P_{u v}} \sum_{q=1}^{Q_{u v}^{p}} \rho_{u v}^{p q} y_{u v}^{p q}\right) \geq \log (1-\varepsilon) .
$$

By (3e), exactly one of the sum elements within the logarithmic function will be nonzero and, hence, this constraint is equivalent to

$$
\sum_{u v \in \mathcal{A}}(\underbrace{\log (1)}_{=0} y_{u v}^{0}+\sum_{p=1}^{P_{u v}} \sum_{q=1}^{Q_{u v}^{p}} \log \left(\rho_{u v}^{p q}\right) y_{u v}^{p q}) \geq \log (1-\varepsilon) .
$$


Note that we can now avoid the use of the slack variables. The problem can be formulated as the following standard ILP model.

$$
\begin{aligned}
& \min \sum_{u v \in \mathcal{A}} \sum_{p=1}^{P_{u v}} \sum_{q=1}^{Q_{u v}^{p}} c_{u v}^{p} y_{u v}^{p q} \\
& \text { s.t. } \sum_{u \in \delta^{-}(v)} x_{u v}^{k}-\sum_{u \in \delta^{+}(v)} x_{v u}^{k}= \begin{cases}-d^{k}, & \text { if } v=s^{k}, \\
d^{k}, & \text { if } v=t^{k}, \\
0, & \text { otherwise }\end{cases} \\
& \sum_{k \in \mathcal{K}} x_{u v}^{k} \leq \sum_{p=1}^{P_{u v}} \sum_{q=1}^{Q_{u v}^{p}} b_{u v}^{p q} y_{u v}^{p q} \\
& \sum_{u v \in \mathcal{A}} \sum_{p=1}^{P_{u v}} \sum_{q=1}^{Q_{u v}^{p}} \log \left(\rho_{u v}^{p q}\right) y_{u v}^{p q} \geq \log (1-\varepsilon) \\
& \sum_{p=1}^{P_{u v}} \sum_{q=1}^{Q_{u v}^{p}} y_{u v}^{p q} \leq 1 \\
& x_{u v}^{k} \geq 0, y_{u v}^{p q} \in\{0,1\} \quad \forall u v \in \mathcal{A}, \forall k \in \mathcal{K}, p=1, \ldots, P_{u v}, q=1, \ldots, Q_{u v}^{p}
\end{aligned}
$$

The resulting formulation is still a large scale ILP, which is, in general, hard to solve.

\subsection{Budget Constrained Formulation}

The problem formulation (4) aims at minimizing the costs while a certain reliability is guaranteed. Depending on the value of $\varepsilon$, the infeasibility tolerance, many problems may be infeasible. Instead, we could ask, how reliable can the network be if a certain budget $B$ is not exceeded? Hence, an alternative formulation of the problem is the following.

$$
\begin{aligned}
\max & \sum_{u v \in \mathcal{A}} \sum_{p=1}^{P_{u v}} \sum_{q=1}^{Q_{u v}^{p}} \log \left(\rho_{u v}^{p q}\right) y_{u v}^{p q} \\
\text { s.t. } 4 \mathrm{~b},(4 \mathrm{c}), 4 \mathrm{e}, 4 \mathrm{ff} & \\
& \sum_{u v \in \mathcal{A}} \sum_{p=1}^{P_{u v}} \sum_{q=1}^{Q_{u v}^{p}} c_{u v}^{p} y_{u v}^{p q} \leq B
\end{aligned}
$$

Thus, formulation (5) maximizes the reliability of the network while the budget constraint (5c) is fulfilled. Note, the budget constraint is a knapsack constraint, which is why the problem is NP-hard.

\subsection{Dependent Random Variables}

In real world applications, the random variables $\eta_{u v}^{p}$ are usually not independent as, e.g., bad weather conditions influence more than one link at the same time. Nevertheless, we can embed the presented formulation (4) in a Branch-and-Bound framework on the basis of [FP09] via a Benders like decomposition to model the case of dependent random variables as described in the following. 


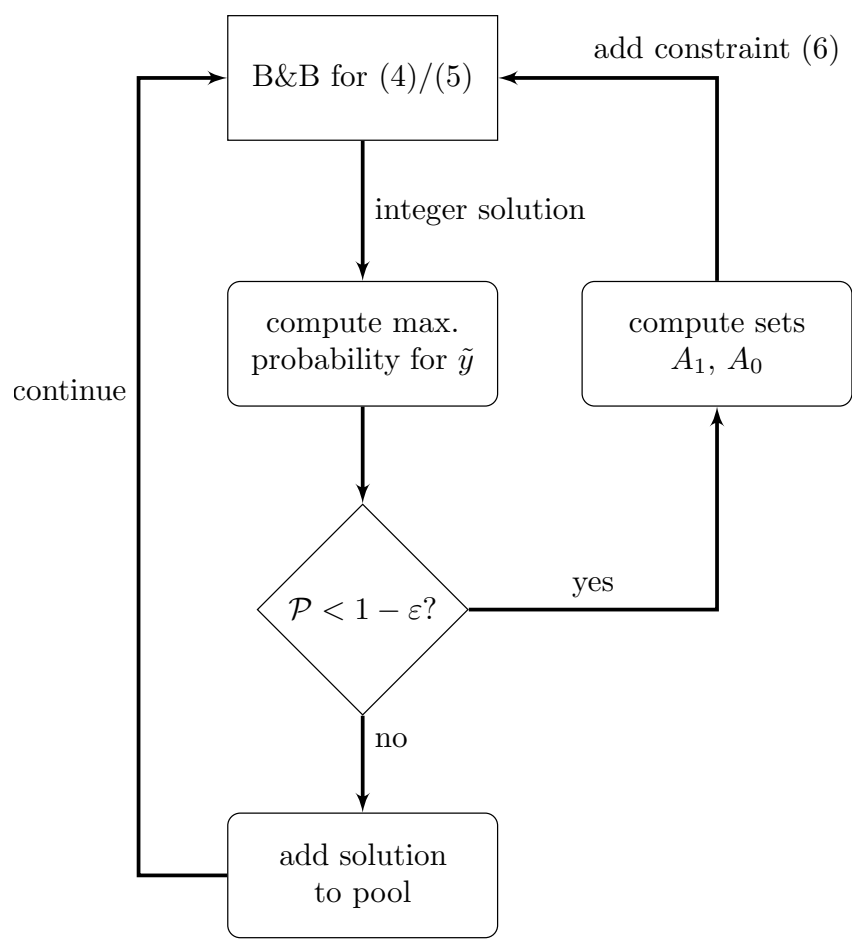

Figure 1: Flowchart of solution framework for dependent random variables.

First, we solve (4) where the probabilities $\rho_{u v}^{p q}$ in constraint (4d) describe the marginal probabilities on a single link. Every integer solution found during the Branch-and-Bound process is then tested for feasibility regarding the actual dependent random variables. This means, we fix the binary decision variables $y$ regarding the computed solution $(\tilde{x}, \tilde{y})$ and determine a corresponding flow that maximizes the probability given in (1c). If the computed probability is less than $1-\varepsilon$, the configuration given by the current values of the decision variables is not part of a feasible solution in case of dependent random variables and hence, the considered solution has to be prohibited. In such a case, we redefine

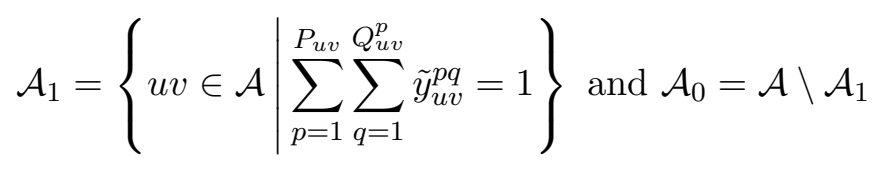

as the sets of installed and non-installed links, respectively. Based on these sets, we add the following constraint as a so-called lazy constraint prohibiting the current solution $(\tilde{x}, \tilde{y})$.

$$
\sum_{u v \in \mathcal{A}_{1}} \sum_{p=1}^{P_{u v}} \sum_{q=1}^{Q_{u v}^{p}} y_{u v}^{p q}+\sum_{u v \in \mathcal{A}_{0}}\left(1-\sum_{p=1}^{P_{u v}} \sum_{q=1}^{Q_{u v}^{p}} y_{u v}^{p q}\right) \leq|\mathcal{A}|-1
$$

Including the new constraint, we continue the Branch-and-Bound routine solving (4). The whole process is depicted in Figure 1 and continues as long as Branch-and-Bound provides new integer solutions.

This framework models random variable dependencies. However, we would like to point out that the computation of the maximum probability for fixed $\tilde{y}$ is typically intractable due to the correlation between the random variables and hence, the framework is difficult to be tested in a computational study. In addition, data with dependent random variables does currently not exist for the considered problem and cannot be computed in a reasonable way. 


\section{Performance Improvements}

The models described in Section 3.3 and 3.4 are very hard to solve. To accelerate the solving process, we present new valid inequalities, so-called cutset inequalities, to improve the dual bound in the first part of this section. To separate these inequalities on the fly, we propose exact separation ILPs. Finally, a primal heuristic to improve the primal bound of the budget constrained model (5) is introduced.

\subsection{Cutset Inequalities}

Constraints (4b), (4c), and (4e) define a classical network design problem studied intensively in the literature [BG96; BCGT98; MMV93; MMV95, RKOW11]. To enhance the performance of ILP solvers, several valid inequalities have been introduced, in particular so-called cutset-based inequalities, exploiting knowledge about the required capacity on a cut in the network.

Let $\mathcal{S} \subset \mathcal{V}$ be a proper and nonempty subset of the node set $\mathcal{V}$ and $\overline{\mathcal{S}}=\mathcal{V} \backslash \mathcal{S}$ its complement. The set $\mathcal{A}(\mathcal{S}, \overline{\mathcal{S}}):=\{u v \in \mathcal{A}: u \in \mathcal{S}, v \in \overline{\mathcal{S}}\}$, i.e., the set of arcs that connect a node in $\mathcal{S}$ to a node in $\overline{\mathcal{S}}$, defines a cutset. Similarly, let $\mathcal{K}(\mathcal{S}, \overline{\mathcal{S}}):=\left\{k \in \mathcal{K}: s^{k} \in \mathcal{S}, t^{k} \in \overline{\mathcal{S}}\right\}$ be the set of demands originating in $\mathcal{S}$ and terminating in $\overline{\mathcal{S}}$. Finally, let $d(\mathcal{S}, \overline{\mathcal{S}}):=\sum_{k \in \mathcal{K}(\mathcal{S}, \overline{\mathcal{S}})} d^{k}$. An appropriate aggregation of constraints $(4 \mathrm{~b}), 4 \mathrm{c})$, and nonnegativity of the variables results in the following base cutset inequalities.

$$
\sum_{u v \in \mathcal{A}(\mathcal{S}, \overline{\mathcal{S}})} \sum_{p=1}^{P_{u v}} \sum_{q=1}^{Q_{u v}^{p}} b_{u v}^{p q} y_{u v}^{p q} \geq d(\mathcal{S}, \overline{\mathcal{S}}) \quad \forall \mathcal{S} \subset \mathcal{V}
$$

These inequalities denote that there should be enough capacity on the arcs of any cutset in order to satisfy the demands that must be routed through it. Base cutset inequalities are necessary for a capacity vector to be feasible, but it is well-known that they are not sufficient in general [CCG09]. By applying Chvátal-Gomory (CG) rounding to base cutset inequalities (cf. [Wol98]), we obtain the well-known cutset inequalities

$$
\sum_{u v \in \mathcal{A}(\mathcal{S}, \overline{\mathcal{S}})} \sum_{p=1}^{P_{u v}} \sum_{q=1}^{Q_{u v}^{p}}\left\lceil\frac{b_{u v}^{p q}}{a}\right\rceil y_{u v}^{p q} \geq\left\lceil\frac{d(\mathcal{S}, \overline{\mathcal{S}})}{a}\right\rceil \quad \forall \mathcal{S} \subset \mathcal{V}
$$

where $a \in\left\{b_{u v}^{p q}: u v \in \mathcal{A}(\mathcal{S}, \overline{\mathcal{S}}), p=1, \ldots, P_{u v}, q=1, \ldots, Q_{u v}^{p}\right\}$. In general, the LP relaxation of (4) does not satisfy (8) although all integer solutions have to satisfy it (cf. [RKOW11).

A novel class of valid inequalities are shifted cutset inequalities which we can obtain from the base cutset inequalities by shifting the coefficients first before applying CG-rounding. Given a cutset $\mathcal{A}(\mathcal{S}, \overline{\mathcal{S}})$ and for $u v \in \mathcal{A}(\mathcal{S}, \overline{\mathcal{S}})$, let $a_{u v}:=\min _{p \in\left\{1, \ldots, P_{u v}\right\}} \min _{q \in\left\{1, \ldots, Q_{u v}^{p}\right\}} b_{u v}^{p q}$ and $a^{\prime} \in$ $\left\{b_{u v}^{p q}-a_{u v}: u v \in \mathcal{A}(\mathcal{S}, \overline{\mathcal{S}}), p=1, \ldots, P_{u v}, q=1, \ldots, Q_{u v}^{p}\right\} \backslash\{0\}$. Note that the parameter $a_{u v}$ is strictly greater than 0 since we do not consider the slack variable $y_{u v}^{0}$ in this context. Multiplying constraints $4 \mathrm{e}$ by $-a_{u v}$ results in

$$
\sum_{p=1}^{P_{u v}} \sum_{q=1}^{Q_{u v}^{p}}\left(-a_{u v}\right) y_{u v}^{p q} \geq-a_{u v} \quad \forall u v \in \mathcal{A} .
$$

Now, we again take the sum over all arcs $u v$ in constraints (4c), apply constraints (9) and CG-rounding using the notation $a(\mathcal{S}, \overline{\mathcal{S}}):=\sum_{u v \in \mathcal{A}(\mathcal{S}, \overline{\mathcal{S}})} a_{u v}$. Thus, we obtain the shifted cutset 
inequalities

$$
\sum_{u v \in \mathcal{A}(\mathcal{S}, \overline{\mathcal{S}})} \sum_{p=1}^{P_{u v}} \sum_{q=1}^{Q_{u v}^{p}}\left\lceil\frac{b_{u v}^{p q}-a_{u v}}{a^{\prime}}\right\rceil y_{u v}^{p q} \geq\left\lceil\frac{d(\mathcal{S}, \overline{\mathcal{S}})-a(\mathcal{S}, \overline{\mathcal{S}})}{a^{\prime}}\right\rceil \quad \forall \mathcal{S} \subset \mathcal{V} .
$$

Note, the presented cutset inequalities are valid for both formulations (4) and (5).

\subsection{Separation of Cutset Inequalities}

As there exist exponentially many subsets $\mathcal{S} \subset \mathcal{V}$, it is not efficient to add all possible cutset inequalities and shifted cutset inequalities. Hence, we rather generate only violated inequalities on the fly. For that purpose we propose ILPs to separate the most violated (shifted) cutset inequalities for the current LP solution exactly, see, e.g., [FLS10] or [KKR13 for cutset separation in the robust network design problem. A cutset inequality is violated if

$$
\sum_{u v \in \mathcal{A}(\mathcal{S}, \overline{\mathcal{S}})} \sum_{p=1}^{P_{u v}} \sum_{q=1}^{Q_{u v}^{p}}\left\lceil\frac{b_{u v}^{p q}}{a}\right\rceil \tilde{y}_{u v}^{p q}-\left\lceil\frac{d(\mathcal{S}, \overline{\mathcal{S}})}{a}\right\rceil<0,
$$

where $\tilde{y}_{u v}^{p q}$ is the current LP solution and $a \in \mathbb{Z}_{\geq 0}$.

For the exact separation of cutset inequalities, we introduce variables $\alpha_{v}$ indicating whether node $v \in \mathcal{V}$ lies in the sub set $\mathcal{S}$, and variables $\beta_{u v}$ deciding whether $u v \in \mathcal{A}(\mathcal{S}, \overline{\mathcal{S}})$. For simplicity we further define

$$
D:=\frac{\sum_{k \in \mathcal{K}} d_{k} \beta_{s^{k} t^{k}}}{a} .
$$

The exact separation of violated cutset inequalities can be formulated as the following ILP (the minimum in (11c) can be linearized in a standard way).

$$
\begin{aligned}
& \min \sum_{u v \in \mathcal{A}}\left(\sum_{p=1}^{P_{u v}} \sum_{q=1}^{Q_{u v}^{p}}\left\lceil\frac{b_{u v}^{p q}}{a}\right\rceil \tilde{y}_{u v}^{p q}\right) \beta_{u v}-z \\
& \text { s.t. } D \leq z \leq D+\frac{a-1}{a} \\
& \alpha_{u}-\alpha_{v} \leq \beta_{u v} \leq \min \left\{1-\alpha_{v}, \alpha_{u}\right\} \forall u, v \in \mathcal{V} \\
& \alpha_{v}, \beta_{u v} \in\{0,1\} \\
& \forall u, v \in \mathcal{V} \\
& z \in \mathbb{N} \text {. }
\end{aligned}
$$

If the optimal objective value is negative, then a violated cutset inequality is found. The variable $z$ together with constraint $(11 \mathrm{~b})$ determines the rounding of the right hand side of the cutset inequality, where $\frac{a-1}{a}$ depicts a small number. Constraints 11c determine the link between variables $\beta_{u v}$ and $\alpha_{v}$ and $\alpha_{u}$, i.e., $\beta_{u v}=1 \Leftrightarrow \alpha_{u}=1 \wedge \alpha_{v}=0$.

For the exact separation of shifted cutset inequalities, we just restate the objective (11a) as

$$
\min \sum_{u v \in \mathcal{A}}\left(\sum_{p=1}^{P_{u v}} \sum_{q=1}^{Q_{u v}^{p}}\left\lceil\frac{b_{u v}^{p q}-a_{u v}}{a^{\prime}}\right\rceil \tilde{y}_{u v}^{p q}\right) \beta_{u v}-z,
$$

set

$$
D:=\frac{\sum_{k \in \mathcal{K}} d_{k} \beta_{s^{k} t^{k}}-\sum_{u v \in \mathcal{A}} a_{u v} \beta_{u v}}{a^{\prime}}
$$

and replace $a$ in constraint $(11 \mathrm{~b})$ by $a^{\prime}$. 


\subsection{A Primal Heuristic}

To find a good solution fast, we introduce the following heuristic to compute values for the decision variables $y$ based on the current LP solution without modifying the flow variables $x$, see Algorithm 1 .

Based on the current flow values, we compute the best bandwidth-configuration pair for each arc, i.e., the pair for which the flow is satisfied, the cost is as low as possible while the reliability is maximal. If the sum over all costs is lower than or equal to the budget, we have found a feasible solution. However, this cannot be guaranteed.

We experienced that the budget $B$ is not always used completely by the constructed solution. Hence, if there is some budget left, we attempt to improve the new solution by replacing bandwidth-configuration pairs with pairs having a higher reliability and still fulfilling the requirements. Note, we assume a non-decreasing ordering of the bandwidths and consider only larger bandwidths in the improvement step.

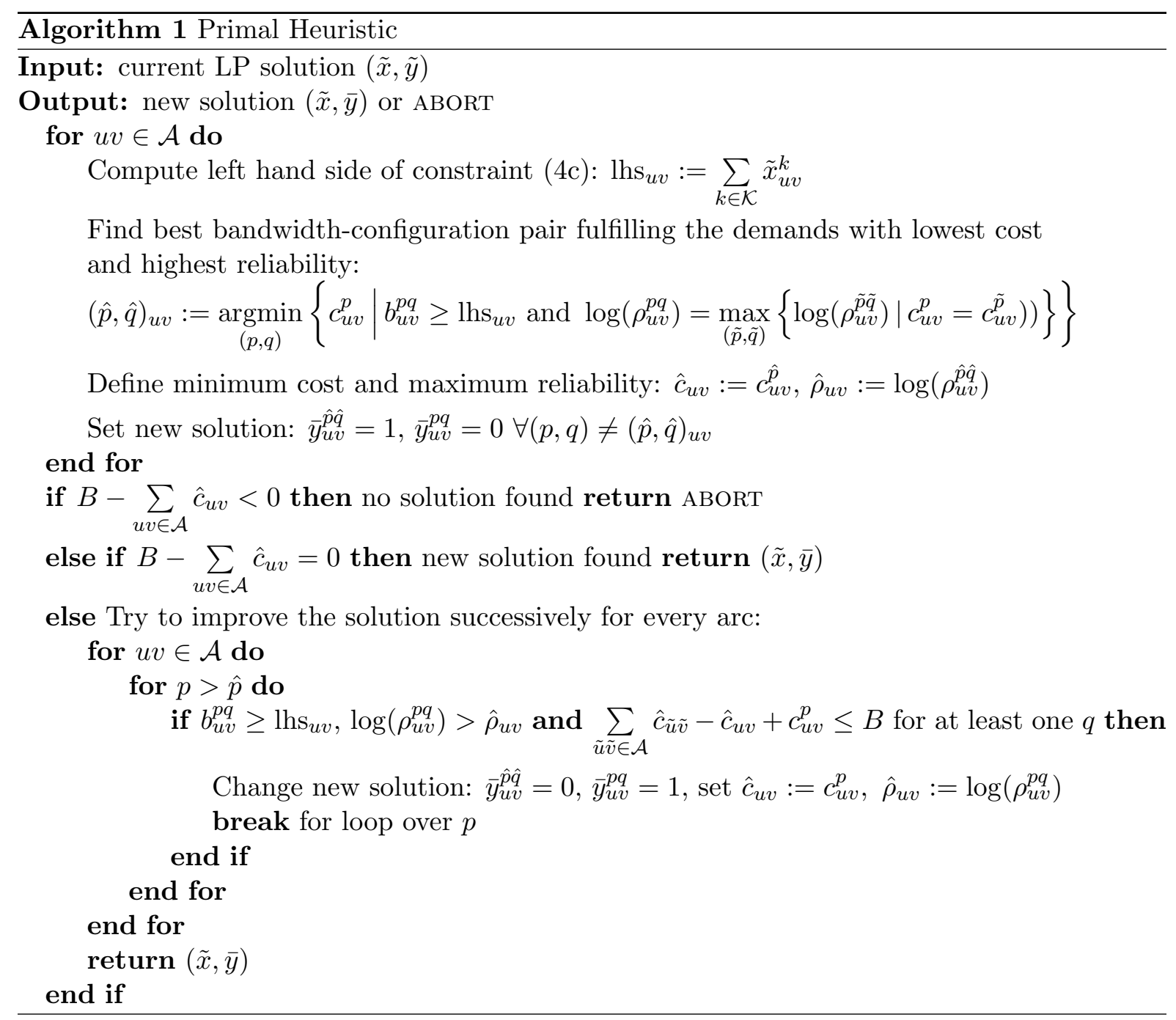




\section{Computational Results}

We have focused our computational study on the case of independent random variables since, for practical instances, the number of scenarios to be considered while using the big- $M$ formulation is unbearable. For the smallest instance studied here, we would have to consider \# configurations $\#$ arcs $\times \#$ bandwidths $=6^{36 \times 3}$ scenarios.

Computations were carried out on a Linux machine with a $3.40 \mathrm{GHz}$ Intel i7-3770 CPU and 32 GB RAM, using IBM ILOG CPLEX 12.4 [IBM12] as underlying solver. A time limit of 2 hours of computation was set for solving each instance, and all other solver settings were preserved at their defaults. Note that CPLEX restricts the number of usable threads to one as soon as a separator is applied.

In this section, we first describe the network topologies and the configurations we used for the considered problem instances. Afterwards, we present on the one hand results on the achievable reliability of the networks with the chance-constrained model compared to models without chance-constraints and on the other hand results on the performance improvements discussed in Section 4

\subsection{Problem Instances}

Given the absence of benchmark instances available in the literature for this problem, we have generated test instances. Network topologies and traffic demands were based on instances from a data library for fixed telecommunication network design, e.g., WDM, SDH, and ATM networks, the Survivable Network Design Library (SNDlib) OPTW10. The selected network topologies studied are shown in Figure 2. These instances were selected to show the potential and limits of our work.

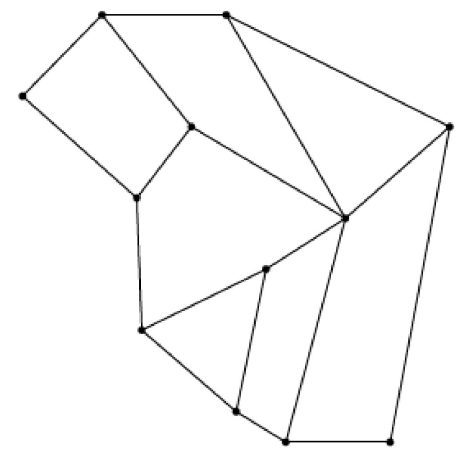

(a) Polska

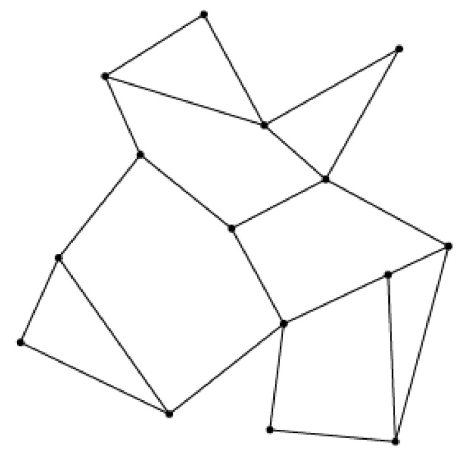

(b) Atlanta

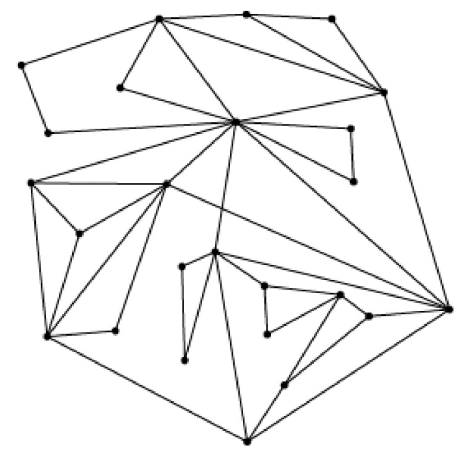

(c) France

Figure 2: SNDlib network topologies.

To fit our application scenario, since microwave links present limited capacity compared to optical fiber, the volumes of traffic demands were rescaled according to a factor $\gamma$, as shown in Table 2, obtained from (12). For each SNDlib instance, observing proportionality of original demands and setting the network reliability at $99 \%$ (i.e., $\varepsilon=0.01$ ), the factor $\gamma$ represents the maximum value for which there exists a feasible flow over the network under the stated probability. 
$\max \gamma$

$$
\begin{aligned}
& \text { s.t. } \sum_{u \in \delta^{-}(v)} x_{u v}^{k}-\sum_{u \in \delta^{+}(v)} x_{v u}^{k}=\left\{\begin{array}{ll}
-d^{k} \gamma, & \text { if } v=s^{k}, \\
d^{k} \gamma, & \text { if } v=t^{k}, \\
0, & \text { otherwise }
\end{array} \quad \forall v \in \mathcal{V}, \forall k \in \mathcal{K}\right. \\
& \quad 4 \mathrm{c}, 4 \mathrm{~d}, 4 \mathrm{de}, 4 \mathrm{ff} \\
& \gamma \geq 0
\end{aligned}
$$

Table 2: Summary of SNDlib problem instances

\begin{tabular}{lrrrr}
\hline Network & $|\mathcal{V}|$ & $|\mathcal{A}|$ & $|\mathcal{K}|$ & $\gamma$ \\
\hline Polska & 12 & 36 & 66 & 0.2252 \\
Atlanta & 15 & 44 & 210 & 0.0170 \\
France & 25 & 90 & 300 & 0.0372 \\
\hline
\end{tabular}

To estimate the probability $\rho_{u v}^{p q}$ and the capacity $b_{u v}^{p q}$ for each arc $u v$, bandwidth choice $p$, and configuration $q$, we have assumed the following radio scenario. We randomly generated the received signal level (RSL) value for each microwave link, assuming a continuous uniform distribution $U(-40,-35)$ (values given in $\mathrm{dBm}$ ). Under this assumption, microwave links typically present very high availability. Based on SNDlib data, path length of microwave links were normalized to a maximum value of $50 \mathrm{~km}$. We have considered 3 frequency bands, $26 \mathrm{GHz}$, $28 \mathrm{GHz}$, and $32 \mathrm{GHz}$, each of them supporting operation at a bandwidth of $7 \mathrm{MHz}, 14 \mathrm{MHz}$, and $28 \mathrm{MHz}$. Note that frequency values are required to estimate the availability of links. But, in this paper, we are not interested in the problem of frequency allocation. (See [AHKMS07] and the references therein for a study of this problem.) For this reason, we have randomly chosen a frequency band among the available choices for each microwave link. Then, supposing Gaussian thermal noise, SNR values for each microwave link and bandwidth were computed.

Furthermore, we have considered six different combinations of modulation and coding, as described in Table 3. The values presented in this table are based on specifications for the WLS500 product by 3Roam [3Ro12]. Then, the capacity $b_{u v}^{p q}$ was computed as the product of the bandwidth and the bandwidth efficiency according to the different radio settings. Finally, the probability $\rho_{u v}^{p q}$ was given by the availability obtained from Vigants-Barnett fading model Bar72. Vig75]. We omit the details for simplicity here.

Table 3: Radio configuration, bandwidth efficiency, and capacity.

\begin{tabular}{lllll}
\hline $\begin{array}{l}\text { Radio } \\
\text { configuration }\end{array}$ & $\begin{array}{l}\text { Bandwidth efficiency } \\
\text { (bps/Hz) }\end{array}$ & $\begin{array}{l}\text { capacity f. } \\
\text { (Mbps) }\end{array}$ & $\begin{array}{l}\text { capacity f. } 14 \mathrm{MHz} \\
\text { (Mbps) }\end{array}$ & $\begin{array}{l}\text { capacity f. } 28 \mathrm{MHz} \\
\text { (Mbps) }\end{array}$ \\
\hline 16-QAM coded & 3.6 & 25.2 & 50.4 & 100.8 \\
16-QAM uncoded & 4.0 & 28 & 56 & 112 \\
64-QAM coded & 5.4 & 37.8 & 75.6 & 151.2 \\
64-QAM uncoded & 6.0 & 42 & 84 & 168 \\
256-QAM coded & 7.2 & 50.4 & 100.8 & 201.6 \\
256-QAM uncoded & 8.0 & 56 & 112 & 224 \\
\hline
\end{tabular}

To normalize our computational results, since prices vary on a country-by-country basis, we have adopted a monetary cost of $1 \$$ per $1 \mathrm{MHz}$ of bandwidth and, therefore, observing that 
spectrum price is usually a linear function of the amount of spectrum with which a license is associated. Note that, under this premise, bandwidth utilization and costs can be used interchangeably.

For each network, we detect a range of reasonable values for the budget $B$. We set the budget interval for Polska to [644, 840], where 644 is the lowest possible value. For a budget less than 644 , the problem is infeasible since we cannot install enough capacity on the arcs. Beyond the budget of 840 , the behaviour changes only very marginally, see Section 5.2 . For Atlanta and France, similar arguments lead to the intervals $[749,1057]$ and $[1414,2002]$, respectively. Due to the possible bandwidth values of 7,14 or $28 \mathrm{MHz}$, we consider budgets by a step of 7 .

\subsection{Reliability Analysis}

In this subsection, based on the reliability of the network topologies, we compare the budget constraint formulation (5) to two formulations without outage probability constraints of the form $(1 \mathrm{c})$.

First, we consider (5) with only one radio configuration available, which is the most natural way to simplify the chance-constraint, i.e., no adaptive modulation and coding (AMC). For all three instances and bandwidth choices, the chosen radio configuration must be the highest one, 256-QAM uncoded, since the problems become infeasible for configurations with lower modulation on all arcs. Thus, the model is limited to the bandwidth selection at all links such that the total traffic requirement is fulfilled. Note that by selecting a single radio configuration, the solution value is a lower bound on the actual network reliability when AMC is employed. In a postprocessing step, for every link $u v$ and the bandwidth $p$ chosen in the solution, we compute the lowest configuration $q$ for which the capacity is sufficient. The actual network reliability now is the product of the corresponding link probabilities $\rho_{u v}^{p q}$.

For Polska and a budget interval of [644,840], Figure 3 displays the reliabilities realized by the budget constraint formulation (5), by the described restricted model (lower bound), and by the postprocessing. The lower bound of the restricted model is between $98.77 \%$ and $98.92 \%$

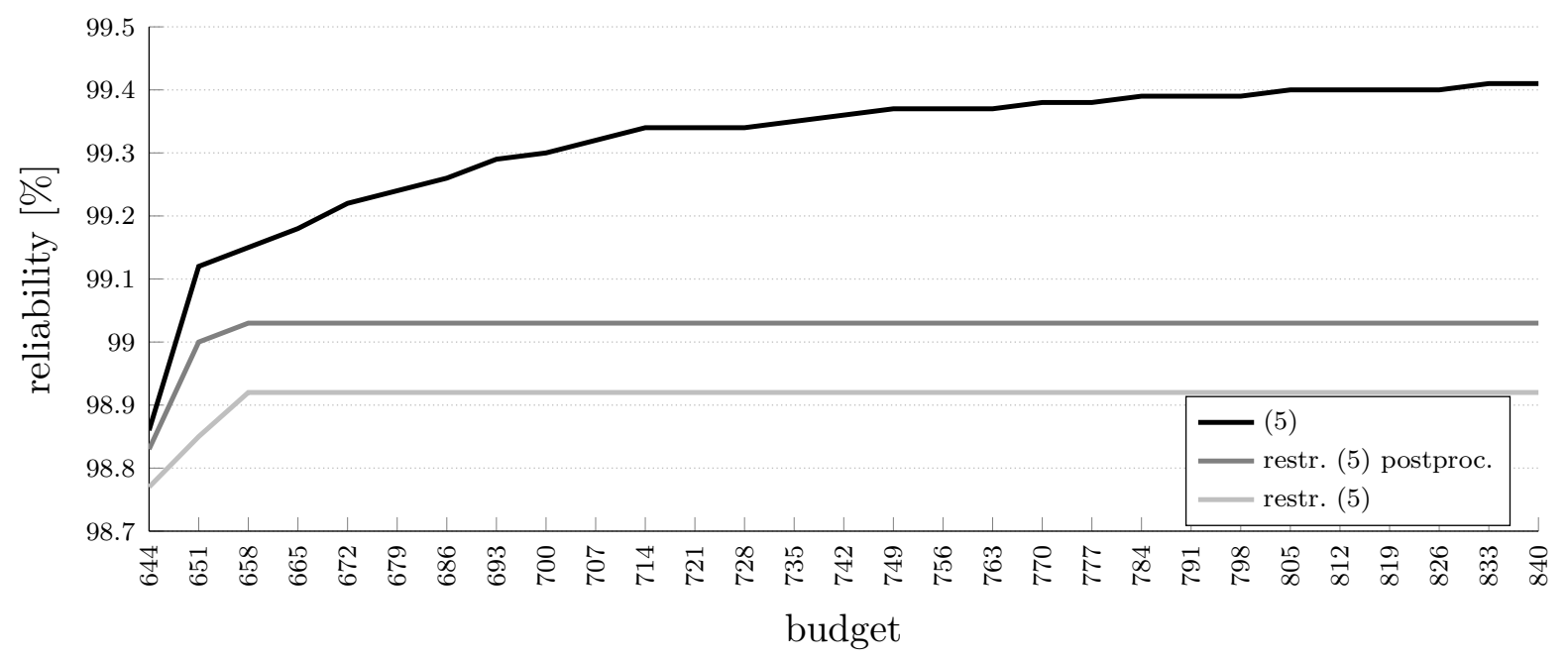

Figure 3: Network reliability for Polska considering only one configuration with/without postprocessing or (5) for different budgets.

and remains constant for budgets greater than or equal to 658. By adaptive modulation and coding, the reliability increases to $98.83 \%$ and $99.03 \%$, respectively. In contrast, we can realize a 
reliability of at least $98.86 \%$ by considering several radio configurations with outage probabilities in formulation (5) and for an increasing budget value, also the reliability of the network is increased until the highest possible reliability of $99.41 \%$ for $B=840$. Note, also budgets greater than 840 are possible but the reliability cannot be increased any further.

Figure 4 presents the results for Atlanta with a budget interval $[749,1057]$ and Figure 5 for France with a budget interval [1414,2002]. In these figures, we additionally display the dual bounds since some/all problems could not be solved to optimality within the time limit. The results for Atlanta are comparable to the results for Polska. The lower bound on the reliability lies between $97.67 \%$ and $97.86 \%$ and the reliability lies between $98.28 \%$ and $98.47 \%$ after postprocessing. The fluctuations in the postprocessed solution values are due to different routings, even in case of equal bandwidth choices. Again, these results are outperformed by the novel chance-constrained model (5) with achievable reliabilities between $98.45 \%$ and $99.06 \%$.

For France, the use of adaptive modulation and coding with the restricted model seems to be less effective. The lower bound lies between $97.8 \%$ and $97.91 \%$ for the restriction to one configuration, where AMC adds at most $0.23 \%$. The highest reliability possible for France with formulation (5) is $99.25 \%$; about $1.18 \%$ higher than with the restricted model after postprocessing.

For networks of the size of France, the budget constraint formulation (5) is harder to solve, in particular, for more restrictive budgets. The first feasible solution we could find within the time limit is $98.78 \%$ for a budget of 1470 . The next solution could then be computed for a budget 1498, which is why the corresponding curve starts at 1498 where all others start at 1414 . Furthermore, many problems could not be solved to optimality leading to fluctuating curves in Figure 5. In general, for higher budgets the solutions are very close to optimal.

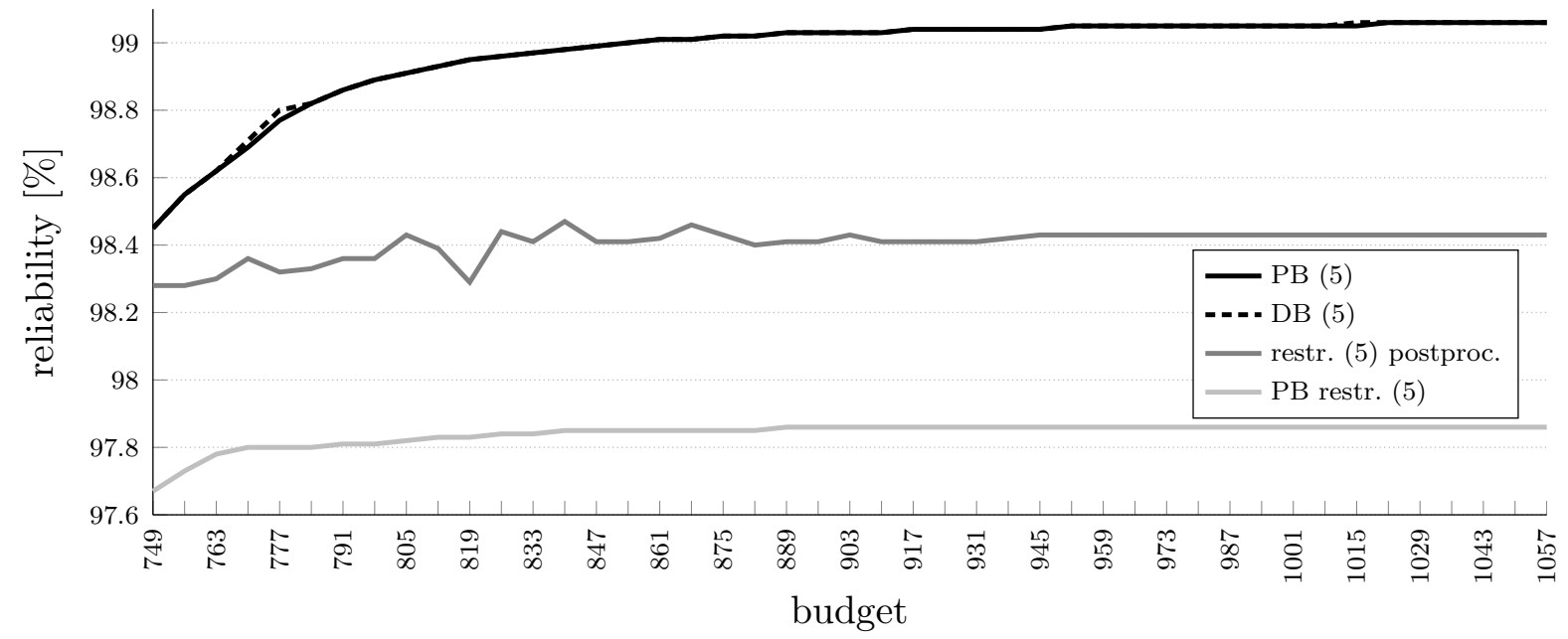

Figure 4: Network reliability for Atlanta considering only one configuration or (5) for different budgets.

The presented results illustrate the significant advantage of the chance-constrained model over the restricted model: we gain higher network reliabilities with reasonable computational effort.

Since the achievable reliabilities for only one possible configuration might be too low in practice, engineers might prefer a different strategy to configure the network. Instead of selecting a single radio configuration for the whole network, one might select a configuration for every arc/bandwidth combination. A reliability of at least, e.g., $99 \%$ can be achieved by requiring 


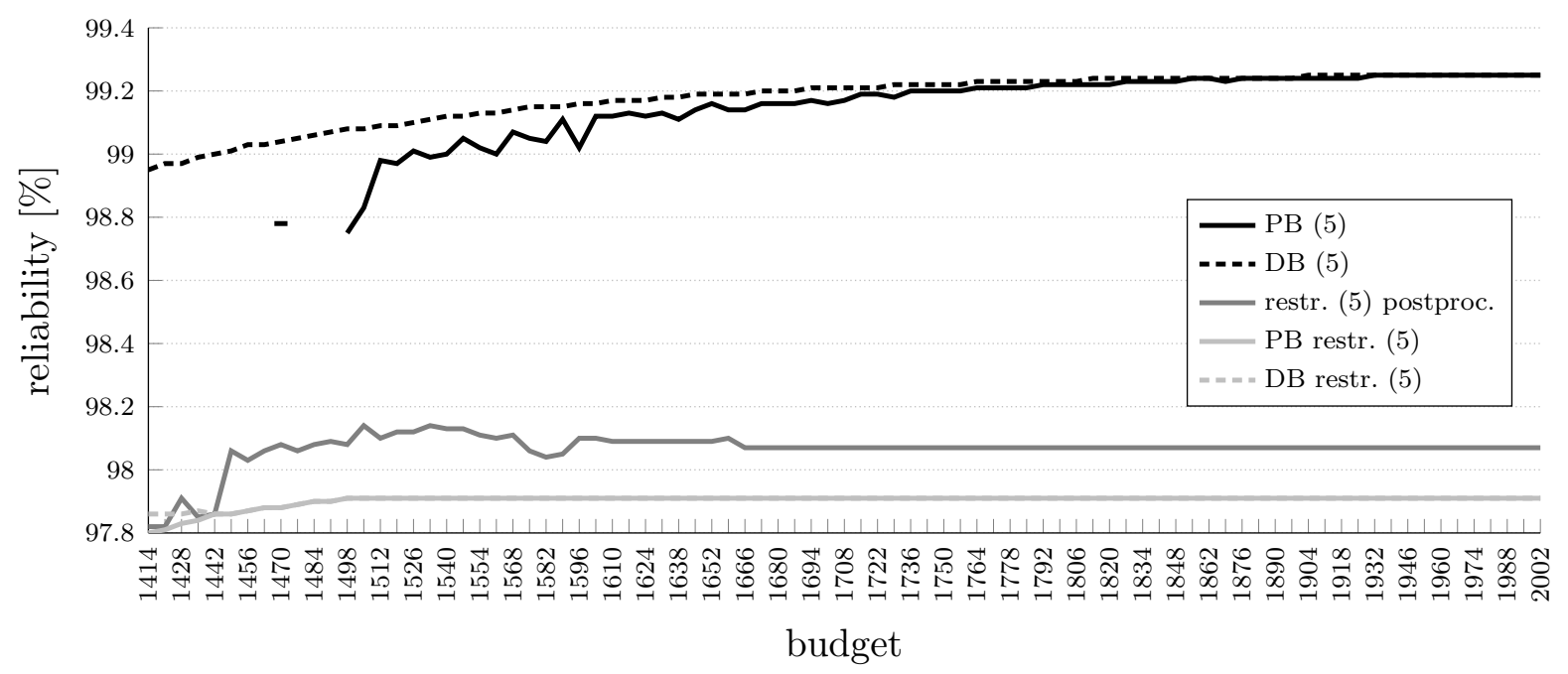

Figure 5: Network reliability for France considering only one configuration or (5) for different budgets.

an uniform minimum probability for all links: $(0.99)^{\frac{1}{\mathcal{A A}}}$. Since the lowest modulation typically has a very high cumulative probability, this minimum probability is achievable for every arc and bandwidth choice. We select the highest configuration satisfying the minimum probability. This approach basically boils down to the model with individual chance constraints for all arcs, see [CCKN11b]. However, a disadvantage is that if we now solve the restricted model (5), the problem becomes infeasible for any budget choice. Thus, not all traffic can be routed in such a configuration. Similar to the choice of $\gamma$, all traffic might be scaled down. If we reduce the traffic requirements from $100 \%$ by steps of $10 \%$, the first percentage resulting in feasible instances for all budgets is $70 \%$ for Polska, and $60 \%$ for Atlanta and France. The highest reliability for Polska with a network load of $70 \%$ is $99.64 \%$, for Atlanta with $60 \%$ network load it is $99.70 \%$ and for France $99.69 \%$. Consequently, we exceed the required reliability of $99 \%$ clearly but for the price of routing less traffic (only $70 \%$ or $60 \%$, respectively) through the networks. In contrast, the clear benefit of formulation (5) is that $100 \%$ of the traffic can be routed with a higher reliability than the required $99 \%$.

\subsection{Analysis of Valid Inequalities and Primal Heuristic}

A part of the results in the previous subsection could only be achieved by applying the cutting planes and primal heuristic of Section 4. To show their importance, we study their performance for the three network topologies in this subsection.

We consider four different settings for the solving of the different problems: CPLEX only, CPLEX and the primal heuristic, CPLEX and the valid inequalities, and CPLEX, the primal heuristic and the valid inequalities. Note, cutset inequalities are separated only in the root node of the branch-and-bound tree via the auxiliary ILP presented in Section 4.1. Additionally, the primal heuristic is applied with a frequency of 20 , i.e., the heuristic is called in every 20th node of the branch-and-bound tree.

For Polska, Figure 6 presents the time reduction for the different settings per budget as well as the CPU times by CPLEX (second axis). For the precise solving times, see Table 6 in the 


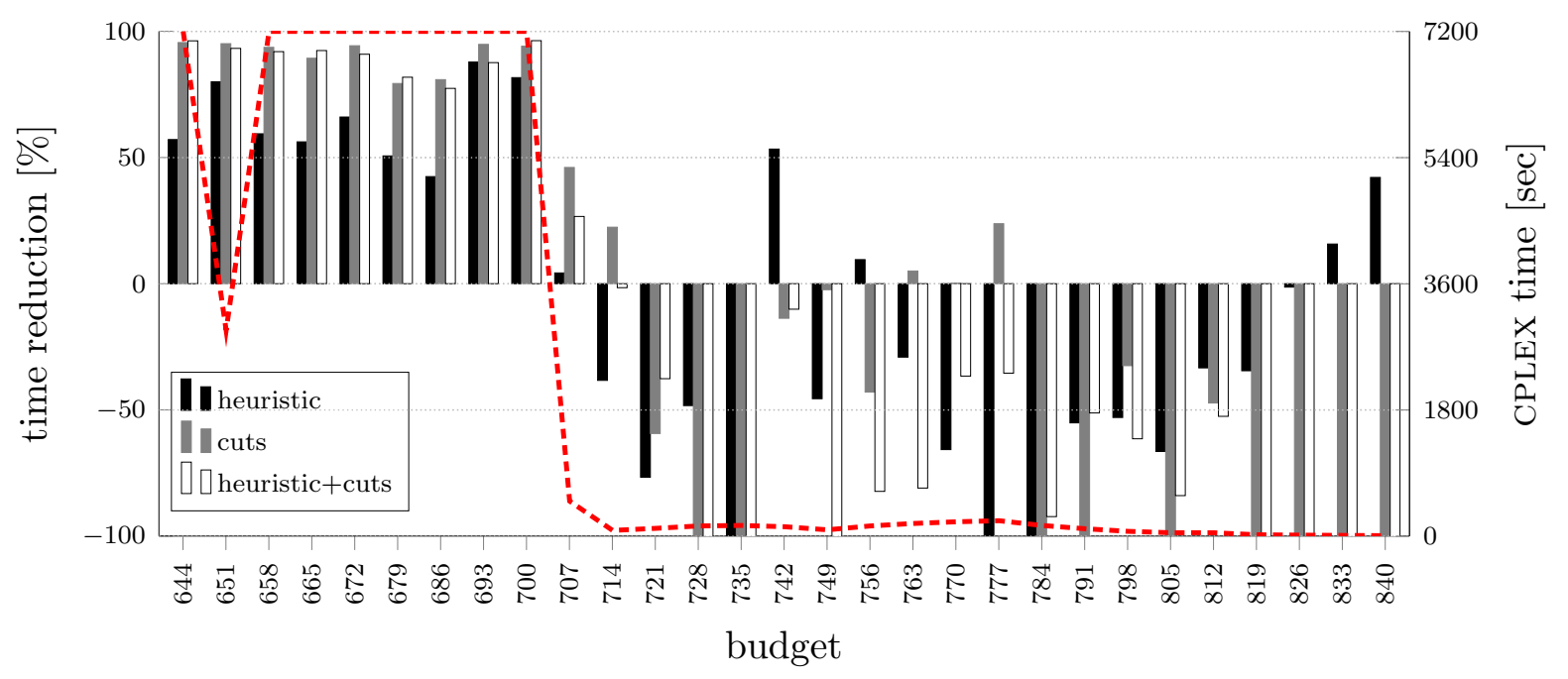

Figure 6: Reduction of computation times for Polska considering different settings and budget values (first y-axis) and absolute times used by CPLEX (second y-axis).

appendix. We compute the time reduction as follows:

$$
\frac{\text { CPLEX time }- \text { advanced time }}{\text { CPLEX time }},
$$

i.e., a value of $20 \%$ means that we can reduce the solving time by $20 \%$ due to the application of the cuts/the primal heuristic compared to the time needed when using CPLEX only, while a value of $-20 \%$ says that we are $20 \%$ slower than CPLEX. Note, if CPLEX exceeds the time limit, the computed time reduction is just a lower bound. Hence, the cuts and the primal heuristic can give a time reduction of at least the computed values if CPLEX reaches the time limit. For readability we set the lowest y-axis value to $-100 \%(+100 \%$ is the highest time reduction possible).

In Figure 6, we display the time reduction for Polska. For a budget $B$ between 644 and 700 excluding 651, the problems are harder to solve and could not be solved within the time limit by CPLEX only, whereas these problems could be solved applying either the primal heuristic or the cutset inequalities. Hence, the time reduction for these problems is high, up to $96.30 \%$. The time reduction achieved by the cutset inequalities is usually higher than by the primal heuristic. For most budget values $B \geq 707$ CPLEX consumes significantly less time. This is why the inequalities and the primal heuristic in most cases cannot reduce the time for these problems.

For Atlanta, the medium-sized network topology, the time reductions and the CPLEx time consumption are displayed in Figure 7. Details can be found in Table 7 in the appendix. The first thirteen problems are the most difficult problems and could not be solved within the time limit in most settings. The most promising setting is the combination of cutset inequalities and the heuristic. Compared to Polska, significantly more problems could be improved by means of cutset inequalities and the application of the primal heuristic.

For a more detailed analysis in the case when all settings exceeded the time limit, we compare the times and the optimality gap for $B=812$ in Figure 8 exemplarily. When using only CPLEX, we could not compute a primal bound within the time limit and hence, also no optimality gap. In contrast, the first gaps computed when separating cuts and/or applying the primal heuristic are below $12 \%$ and are found between 18 (heuristic) and $270 \mathrm{sec}$ (cuts). Due to the 


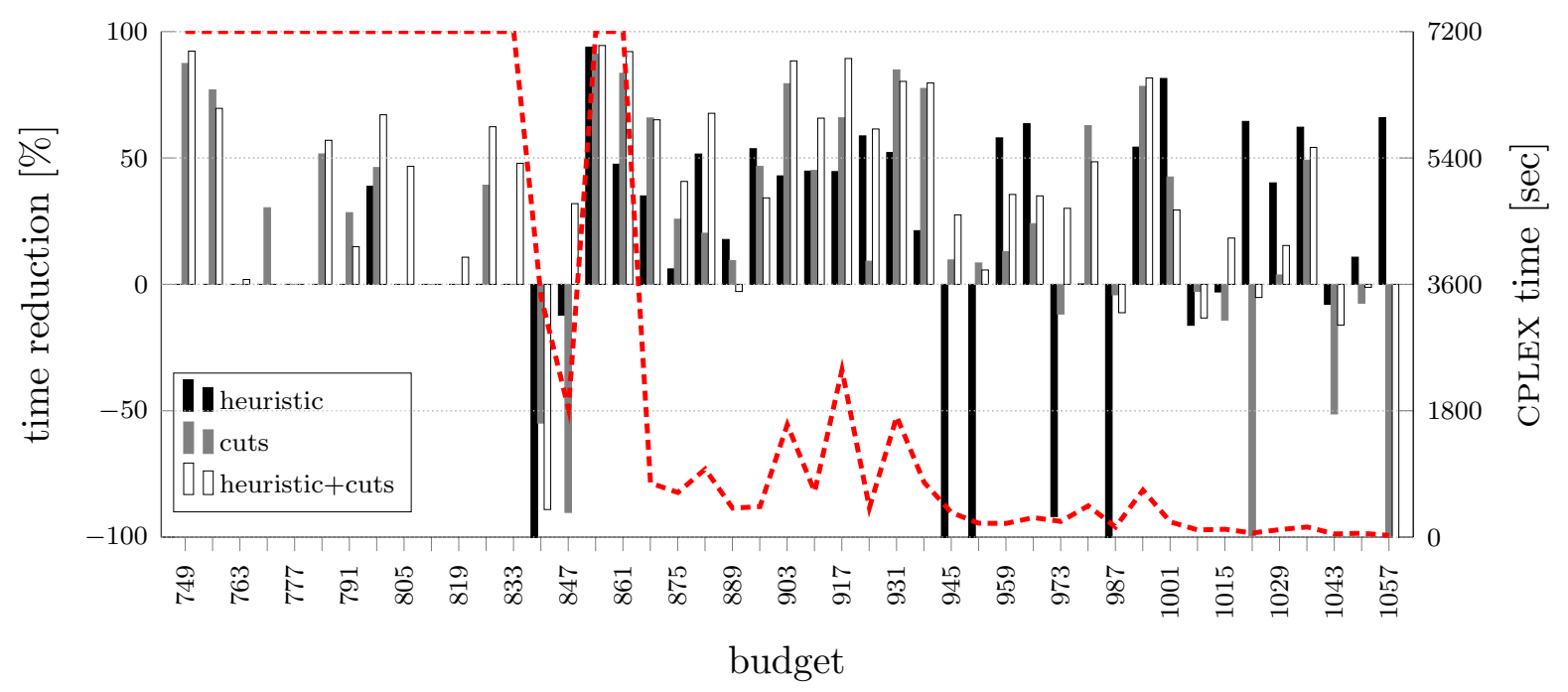

Figure 7: Reduction of computation times for Atlanta considering different settings and budget values (first y-axis) and absolute times used by CPLEX (second y-axis).

separation of cutset inequalities, more time is needed to compute a first primal bound which is why applying just the heuristic is the first setting computing an optimality gap. When applying cutset inequalities and the primal heuristic, we are noticeably earlier below $5 \%, 2 \%$ and $1 \%$ optimality gap than with the other two settings, see also Table 4 , and the best gap $(0.44 \%)$ is also computed by the combination of cutset inequalities and the heuristic. Hence, also for an instance which cannot be solved to optimality within the time limit, this setting gives the best result.

Table 4: Times (in sec) when optimality gap is less than a certain percentage for Atlanta considering the different settings and budget $B=812$.

\begin{tabular}{cccc}
\hline gap & CPLEX + heuristic & CPLEX + cuts & CPLEX + heuristic + cuts \\
\hline $5 \%$ & 356.46 & 686.33 & 317.69 \\
$2 \%$ & 4056.85 & 1951.13 & 1386.84 \\
$1 \%$ & -- & 4927.09 & 3921.74 \\
\hline
\end{tabular}

Finally, we evaluate the results for France where we fix the budget to the interval $[1414,2002]$. As no problem could be solved to optimality for neither setting, we consider the optimality gaps reached after two hours instead of the times, see Table 8 in the appendix for the complete results. Hence, we compute the gap reduction as

$$
\frac{\text { CPLEX gap }- \text { advanced gap }}{\text { CPLEX gap }},
$$

i.e., a value of $20 \%$ means that we can reduce the gap by $20 \%$ due to the application of the cuts/the primal heuristic compared to the gap found when using CPLEX only, while a value of $-20 \%$ says that we increased the gap by $20 \%$ compared to CPLEX. If no primal bound could be found, we set the gap to $100 \%$. Hence, the given values are again the lower bounds. For readability, we once more scale from $-100 \%$ to $100 \%$ although the gap can be increased by more than $100 \%$. 


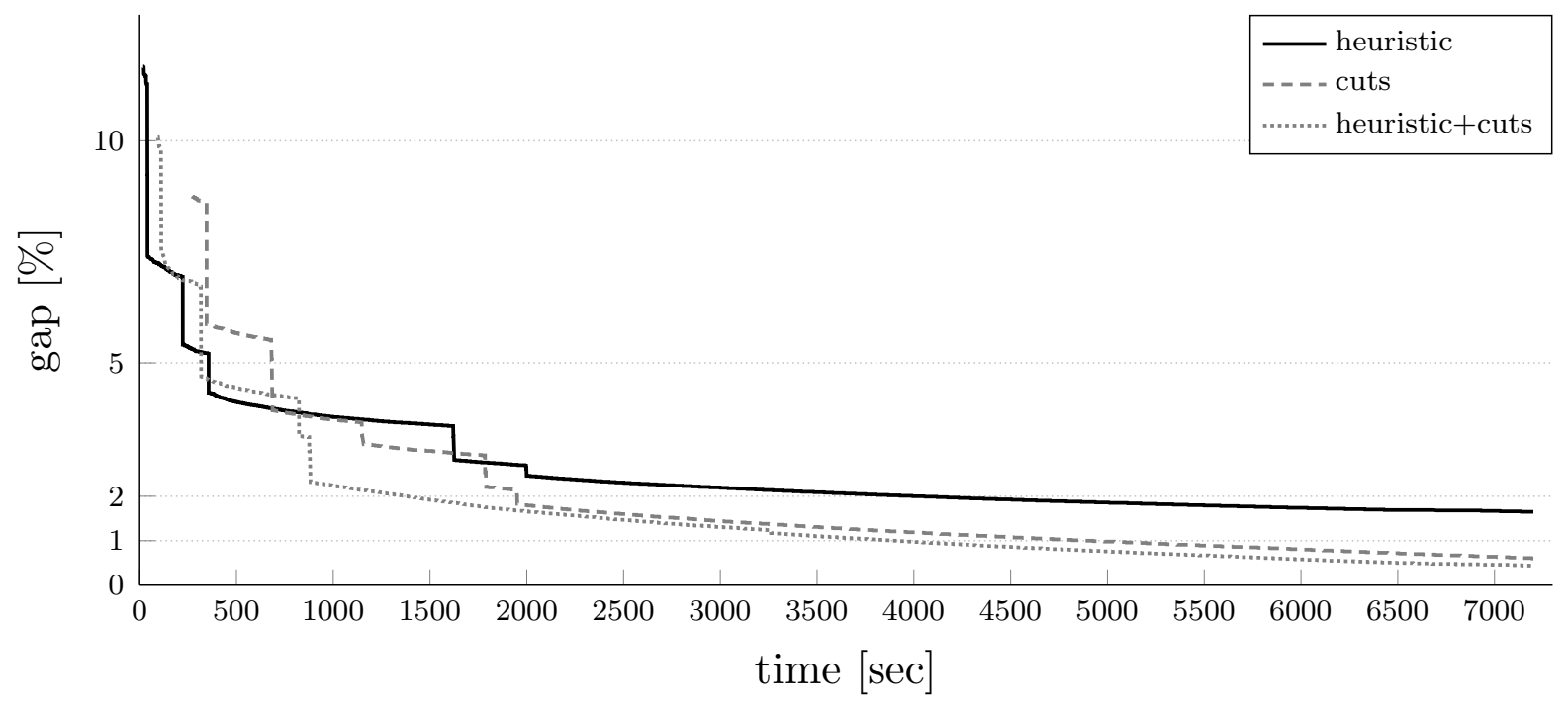

Figure 8: Integrality gap per time for Atlanta considering different settings and budget value $B=812$.

For better readability, we split the figure for the gap reduction into two figures, Figure 9 for budgets in $[1414,1736]$ and Figure 10 for budgets in $[1743,2002]$. Since not a single solution could be found for $B \in[1414,1463]$, we start with $B=1470$ in Figure 9. For almost all problems, the optimality gap could be reduced significantly when separating the cutset inequalities and applying the primal heuristic. Just for the easier problems with a budget greater than 1848 CPLEX has already quite low gaps, which we could not decrease. Since the cutset inequalities just improve the dual bound, a primal bound is usually found later. This is the reason why the gaps can be higher when only the valid inequalities are separated.

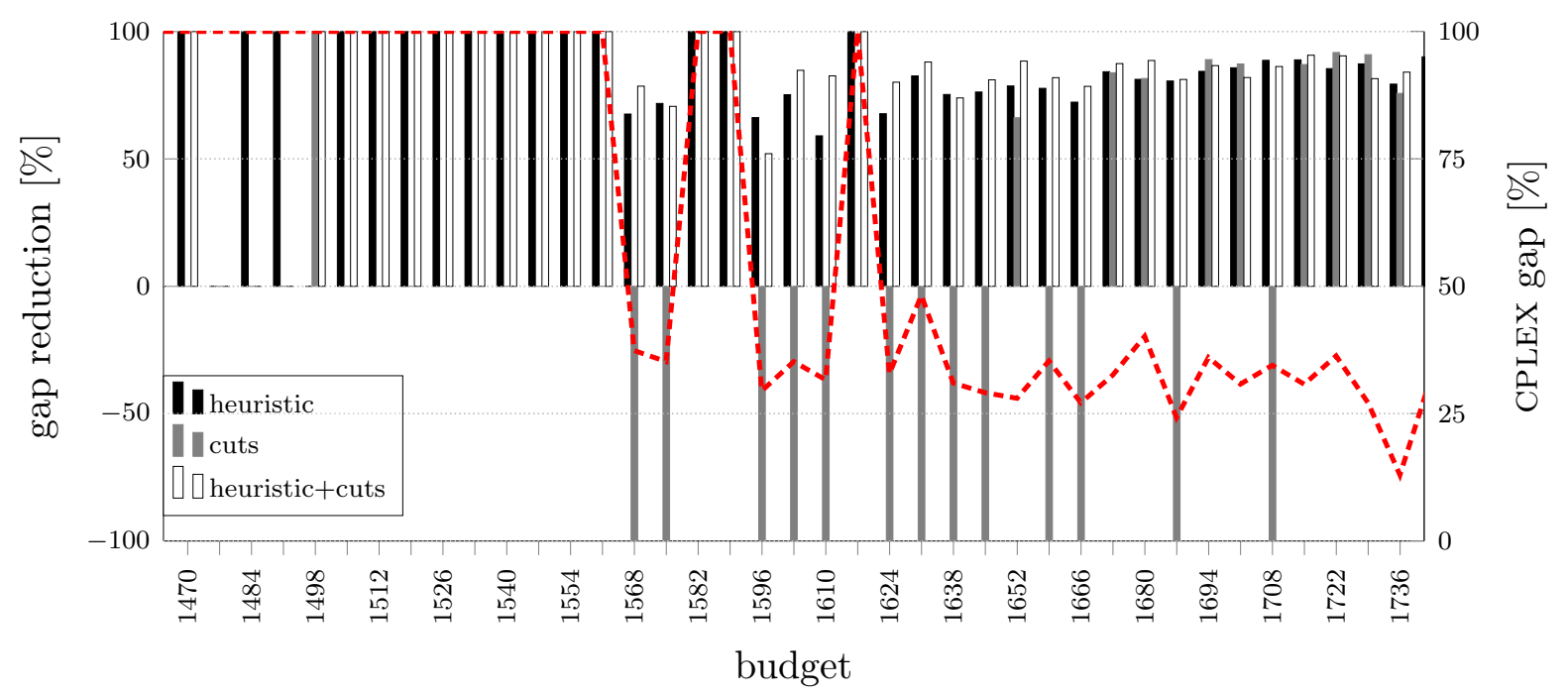

Figure 9: Reduction of optimality gaps for France considering different settings and budget values in $[1414,1736]$ (first y-axis) and original gaps computed by CPLEX (second y-axis).

The gap reduction by the heuristic (with or without cutting planes) is dramatic, showing the 


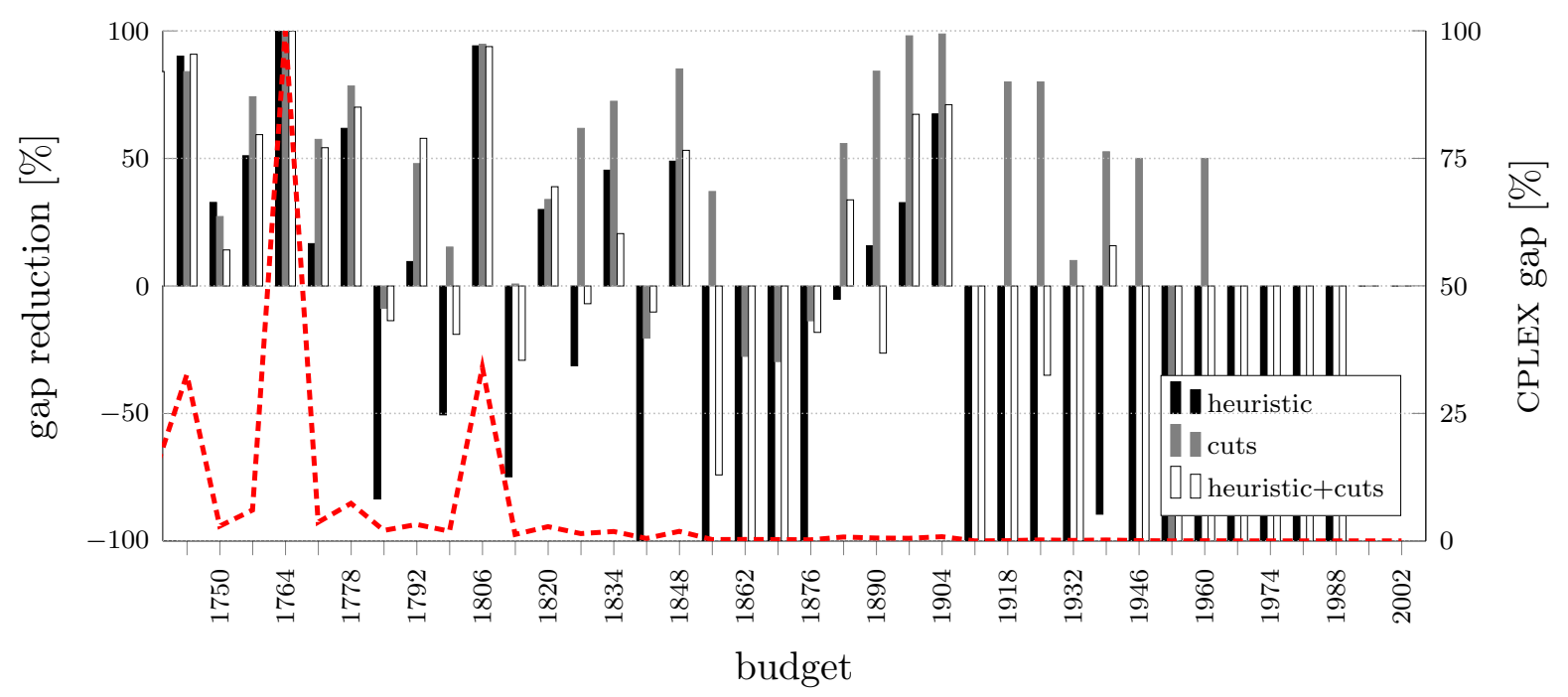

Figure 10: Reduction of optimality gaps for France considering different settings and budget values in $[1743,2002]$ (first y-axis) and original gaps computed by CPLEX (second y-axis).

importance of this relative simple idea. To understand its effectiveness, we revisit the primal heuristic once again briefly but from different perspectives, e.g., by regarding the impact on the actual solutions found. Table 5 displays all considered aspects and the used budget intervals for the three network topologies. Based on the intervals, the number of test instances differs per

Table 5: Effectiveness of primal heuristic with respect to different aspects.

\begin{tabular}{lccc}
\hline & $\begin{array}{c}\text { Polska } \\
{[644,840]}\end{array}$ & $\begin{array}{c}\text { Atlanta } \\
{[749,1057]}\end{array}$ & $\begin{array}{c}\text { France } \\
{[1414,2002]}\end{array}$ \\
\hline \# instances & 29 & 45 & 85 \\
\# inst. first sol. by heur. (in \%) & $23(79.3 \%)$ & $42(93.3 \%)$ & $75(88.2 \%)$ \\
\# inst. no sol. found without heur. & - & 11 & 26 \\
\# inst. no sol. found with heur. (in \%) & - & $2(18.18 \%)$ & $10(38.5 \%)$ \\
absolute increase in primal bound & $0.2 \%$ & $0.6 \%$ & $2.8 \%$ \\
\% of time used to find first sol. with heur. & $45.0 \%$ & $13.6 \%$ & $23.7 \%$ \\
best solution found by heuristic & 1 & 24 & 1 \\
\hline
\end{tabular}

network. For Polska, the first solution that could be found is computed by the primal heuristic in 23 of 29 cases, which corresponds to $79.3 \%$ of all instances. For Atlanta and France, this is the case for 42 out of 45 (93.3\%) instances and 75 out of 85 (88.2\%), respectively. Note, for the remaining instances of Atlanta and France, no primal solution could be found at all within the time limit. The number of such instances can be reduced from 11 to 2 for Atlanta and from 26 to 10 for France by the application of the presented primal heuristic. Hence, even for the more complex network topologies, the proposed heuristic is absolutely effective in finding a feasible solution.

Moreover, comparing the values of the first primal solutions found with and without the heuristic - only for those cases where a primal solution could be computed with both settings, the absolute values found by the heuristic (given as a percentage) are usually larger than the values found without the heuristic. On average, the probability is increased by $0.2 \%$ for 
Polska, $0.6 \%$ for Atlanta and $2.8 \%$ for France. Thus, the larger the network topology, the more the first primal bound found can be improved by the primal heuristic.

For the medium-sized network Atlanta, in more than half of the instances (24) the optimal solution is found by the heuristic, whereas this is only once the case for Polska. Also for France, the best known solution is found once by the heuristic. But remember that in this case, the heuristic works as an accelerator, allowing CPLEX to find better primal solutions (at all).

Finally, regarding the computation times until the first solution is found, the primal heuristic uses on average only $45.0 \%, 13.6 \%$ and $23.7 \%$ of the time spent without the heuristic for Polska, Atlanta and France, respectively. The speed-up is implicitly also included in Figures 6 and 7 but not as considerably as in the numbers of Table 5 since the speed-up until the first solution is found is not necessarily conveyed to the end of the solution process.

In summary, the results of this section demonstrate the gains of both the valid inequalities and the primal heuristic, and especially of their combination. For larger instances, these add-ons are indispensable for close-to-optimal solutions in a reasonable time.

\section{Conclusion}

In this paper, we have presented a chance-constrained programming approach to tackle the problem of assigning bandwidths for reliable fixed point-to-point wireless networks under uncertain radio configurations. We have introduced ILP formulations for this problem including a budget constrained model. To improve the performance, we introduced valid inequalities, exact separation by ILP and a primal heuristic. The computational study revealed the gains of the valid inequalities as well as the effectiveness of the primal heuristic. Furthermore, we investigated the reliability of various network topologies for different budget values and compared the budget constrained model to two alternative formulations which do not incorporate the joint outage probability constraint. The results show a significant gain in reliability by the joint probability model, though solving times increase.

As future work, we intend to model this problem as a tri-level two-player game [CCP11], where the network operator decides the bandwidth assignment, then external random factors cause the deterioration of the performance of some links, and finally, the network operator attempts to find a feasible flow over the residual capacity of the network. In fact, bandwidth assignment and network flow decisions take place in different time and, therefore, we can hopefully save bandwidth utilization allowing dynamic routing. In addition, to improve the reliability of the network, we envisage a study on the impact of traffic fluctuations.

\section{Acknowledgments}

This paper results from a research cooperation which was promoted by the PROCOPE program, a bilateral program funded by the German Academic Exchange Service (DAAD) and by the French Ministry of Foreign Affairs. Additionally, this work has been supported by the Villum Kann Rasmussen foundation, ANR DIMAGREEN and ECOSCELLS, Région PACA, and SME 3ROAM, as well as the excellence initiative of the German federal and state governments, the DFG research grant KO 2311/3-1, SCHM 2643/5-1, and by the UMIC Research Centre at RWTH Aachen University. 


\section{References}

[AHKMS07] K. Aardal, S. van Hoesel, A. M. C. A. Koster, C. Mannino, and A. Sassano. Models and solution techniques for frequency assignment problems. Ann. oper. res., 153(1):79-129, 2007.

[And03] H. Anderson. Fixed broadband wireless system design. John Wiley \& Sons, first edition, 2003.

[Bal80] M. O. Ball. Complexity of network reliability computations. Networks, 10(2):153$165,1980$.

[Bal86] M. O. Ball. Computational complexity of network reliability analysis: an overview. Ieee transactions on reliability, 35(3):230-239, 1986.

[Bar72] W. T. Barnett. Multipath propagation at 4, 6 and 11 GHz. Bell system technical journal, 51(2):311-361, 1972.

[BB10] P. Beraldi and M. E. Bruni. An exact approach for solving integer problems under probabilistic constraints with random technology matrix. English. Annals of operations research, 177(1):127-137, 2010. ISSN: 0254-5330. URL: http://dx. doi.org/10.1007/s10479-009-0670-9.

[BCGT98] D. Bienstock, S. Chopra, O. Günlük, and C. Y. Tsai. Minimum cost capacity installation for multicommodity network flows. Math. program., 81(2):177-199, 1998.

[BG96] D. Bienstock and O. Günlük. Capacitated network design - polyhedral structure and computation. Informs journal on computing, 8(3):243-259, 1996.

[BJ88] T. B. Brecht and C. C. J. Lower bounds on two-terminal network reliability. Discrete applied mathematics, 21(3):185-198, 1988.

[BKC01] J. Burns, S. Kirtay, and D. Court. Study on administrative and frequency fees related to the licensing of networks involving the use of frequencies. Technical report (1307/AE/EC/FR/1). European Commission Directorate General Information Society, 2001.

[CCG09] A. M. Costa, J.-F. Cordeau, and B. Gendron. Benders, metric and cutset inequalities for multicommodity capacitated network design. Comput. optim. appl., 42(3):371-392, 2009.

[CCKN11a] G. Claßen, D. Coudert, A. M. C. A. Koster, and N. Nepomuceno. A chanceconstrained model \& cutting planes for fixed broadband wireless networks. In 5th international network optimization conference (inoc 2011). Volume 6701. In LNCS. Springer, 2011, pages 37-42.

[CCKN11b] G. Claßen, D. Coudert, A. M. C. A. Koster, and N. Nepomuceno. Bandwidth assignment for reliable fixed broadband wireless networks. In 12th IEEE international symposium on a world of wireless mobile and multimedia networks (wowmom), 2011, pages 1-6.

[CCP11] R. L. Chen, A. Cohn, and A. Pinar. An implicit optimization approach for survivable network design. In IEEE network science workshop, June 2011, pages 180187. DOI: 10.1109/NSW.2011.6004644.

[CNR10] D. Coudert, N. Nepomuceno, and H. Rivano. Power-efficient radio configuration in fixed broadband wireless networks. Comput. commun., 33(8):898-906, 2010. 
[Cra96] R. K. Crane. Electromagnetic wave propagation through rain. John Wiley \& Sons, 1996.

[DBHRX07] S. Dominiak, N. Bayer, J. Habermann, V. Rakocevic, and B. Xu. Reliability analysis of IEEE 802.16 mesh networks. In 2nd ieee/ifip international workshop on broadband convergence networks, bcn 200\%, 2007, pages 1-12.

[FLS10] M. Fischetti, A. Lodi, and D. Salvagnin. Just mip it! In, Matheuristics. Volume 10, in Annals of Information Systems, pages 39-70. Springer, 2010.

[FP09] B. Fortz and M. Poss. An improved benders decomposition applied to a multilayer network design problem. Operations research letters, 37(5):359 -364, 2009. ISSN: 0167-6377.

[GC97] A. Goldsmith and S.-G. Chua. Variable-rate variable-power MQAM for fading channels. Ieee trans. commun., 45:1218-1230, 1997.

[GC98] A. Goldsmith and S.-G. Chua. Adaptive coded modulation for fading channels. Ieee trans. commun., 46(5):595-602, 1998.

[KKR13] A. M. C. A. Koster, M. Kutschka, and C. Raack. Robust network design: formulations, valid inequalities, and computations. Networks, 61(2):128-149, 2013. ISSN: 1097-0037. DOI: 10.1002/net.21497.

[Klo10] O. Klopfenstein. Solving chance-constrained combinatorial problems to optimality. Comput. optim. appl., 45(3):607-638, 2010.

[LAN10] J. Luedtke, S. Ahmed, and G. L. Nemhauser. An integer programming approach for linear programs with probabilistic constraints. Math. program., 122(2):247$272,2010$.

[Leh10] H. Lehpamer. Microwave transmission networks: planning, design, and deployment. McGraw-Hill, 2010.

[Lit09] S. Little. Is microwave backhaul up to the $4 \mathrm{G}$ task? Ieee microwave magazine, 10(5):67-74, 2009.

[MMV93] T. L. Magnanti, P. Mirchandani, and R. Vachani. The convex hull of two core capacitated network design problems. Math. program., 60(2):233-250, 1993.

[MMV95] T. L. Magnanti, P. Mirchandani, and R. Vachani. Modelling and solving the twofacility capacitated network loading problem. Oper. res., 43(1):142-157, 1995.

[OPTW10] S. Orlowski, M. Pióro, A. Tomaszewski, and R. Wessäly. SNDlib 1.0-Survivable Network Design Library. Networks, 55(3):276-286, 2010.

[PB84] J. S. Provan and M. O. Ball. Computing network reliability in time polynomial in the number of cuts. Operations research, 32(3):516-526, 1984.

[Pré95] A. Prékopa. Stochastic programming. Kluwer, 1995.

[RKOW11] C. Raack, A. M. C. A. Koster, S. Orlowski, and R. Wessäly. On cut-based inequalities for capacitated network design polyhedra. Networks, 57(2):141-156, 2011.

[Rus02] A. Ruszczyński. Probabilistic programming with discrete distributions and precedence constrained knapsack polyhedra. Math. program., 93(2):195-215, 2002.

[SDR09] A. Shapiro, D. Dentcheva, and A. Ruszczyński. Lectures on stochastic programming: modeling and theory. SIAM, 2009.

[Vig75] A. Vigants. Space-diversity engineering. Bell system technical journal, 54(1):103$142,1975$. 
[Wol98] L. Wolsey. Integer programming. John Wiley \& Sons, first edition, 1998.

[3Ro12] 3Roam. 3Roam website. 2012. URL: http://www.3roam.com.

[IBM12] IBM ILOG. CPLEX Optimization Studio 12.4. 2012. URL: http://www-01.ibm. com/software/integration/optimization/cplex-optimizer/.

[ITU12] ITU. The ITU website. 2012. URL: http://www.itu.int/. 


\section{A Online Supplement}

Table 6: Solving times (in sec) for Polska considering the different settings and budgets.

\begin{tabular}{ccccc}
\hline budget & CPLEX & heuristic & cuts & heuristic+cuts \\
\hline 644 & 7200.6 & 3088.06 & 320.21 & 271.2 \\
651 & 2937.38 & 587 & 143.59 & 198.69 \\
658 & 7200.63 & 2923.25 & 457.17 & 579.6 \\
665 & 7200.62 & 3152.94 & 767.95 & 548.74 \\
672 & 7200.6 & 2444.55 & 411.33 & 654.14 \\
679 & 7200.65 & 3554.27 & 1488.02 & 1309.79 \\
686 & 7200.59 & 4144.47 & 1378.53 & 1627.94 \\
693 & 7200.6 & 875.07 & 373.07 & 892.78 \\
700 & 7200.6 & 1320.53 & 425.01 & 266.15 \\
707 & 500.13 & 478.79 & 269.79 & 366.9 \\
714 & 82.18 & 113.67 & 63.77 & 83.48 \\
721 & 111.46 & 196.94 & 177.62 & 153.41 \\
728 & 145.81 & 216.23 & 1180.26 & 409.27 \\
735 & 153.01 & 532.43 & 369.98 & 352.58 \\
742 & 134.98 & 62.97 & 153.48 & 148.62 \\
749 & 90.85 & 132.25 & 93.01 & 215.63 \\
756 & 145.92 & 132 & 208.6 & 266 \\
763 & 181.1 & 233.8 & 171.97 & 327.76 \\
770 & 205.29 & 340.27 & 204.9 & 280.44 \\
777 & 220.61 & 454.66 & 168.18 & 298.81 \\
784 & 152.88 & 422.98 & 342.22 & 293.97 \\
791 & 106.38 & 165.05 & 216.11 & 160.87 \\
798 & 69 & 105.58 & 91.42 & 111.4 \\
805 & 48.97 & 81.53 & 101.03 & 90.09 \\
812 & 48.86 & 65.16 & 71.96 & 74.54 \\
819 & 20.26 & 27.25 & 51.03 & 65.28 \\
826 & 14.76 & 14.96 & 48.71 & 48.61 \\
833 & 10.94 & 9.22 & 44.49 & 36.08 \\
840 & 7.29 & 4.22 & 34.68 & 27.56 \\
\hline & & & &
\end{tabular}


Table 7: Solving times (in sec) for Atlanta considering the different settings and budgets.

\begin{tabular}{|c|c|c|c|c|}
\hline budget & CPLEX & heuristic & cuts & heuristic + cuts \\
\hline 749 & 7200.68 & 7200.81 & 909.3 & 557.46 \\
\hline 756 & 7200.79 & 7200.91 & 1660.72 & 2187.26 \\
\hline 763 & 7200.78 & 7200.83 & 7200.68 & 7062.82 \\
\hline 770 & 7200.78 & 7200.81 & 5018.13 & 7200.7 \\
\hline 777 & 7200.77 & 7200.79 & 7200.81 & 7200.9 \\
\hline 784 & 7200.77 & 7200.78 & 3485.12 & 3095.44 \\
\hline 791 & 7200.78 & 7200.81 & 5157.85 & 6126.28 \\
\hline 798 & 7200.79 & 4407.24 & 3873.42 & 2369.67 \\
\hline 805 & 7200.8 & 7200.8 & 7200.77 & 3839.47 \\
\hline 812 & 7200.77 & 7200.8 & 7200.8 & 7200.78 \\
\hline 819 & 7200.79 & 7200.82 & 7200.78 & 6428.32 \\
\hline 826 & 7200.78 & 7201.29 & 4376.63 & 2711.42 \\
\hline 833 & 7200.78 & 7201.87 & 7200.86 & 3752.65 \\
\hline 840 & 3421.65 & 7201.11 & 5299.32 & 6469.68 \\
\hline 847 & 1828.66 & 2051.27 & 3478.62 & 1244.75 \\
\hline 854 & 7200.77 & 441.48 & 644.83 & 396.64 \\
\hline 861 & 7200.78 & 3777.88 & 1189.83 & 570.58 \\
\hline 868 & 770.73 & 501.01 & 263.53 & 268.99 \\
\hline 875 & 635.25 & 596.47 & 471.58 & 376.55 \\
\hline 882 & 969.03 & 469.36 & 773.11 & 313.24 \\
\hline 889 & 412.7 & 339.43 & 373.95 & 424.37 \\
\hline 896 & 434.47 & 201.33 & 231.77 & 285.85 \\
\hline 903 & 1602.09 & 914.55 & 330.4 & 185.9 \\
\hline 910 & 647.13 & 357.17 & 355.49 & 221.71 \\
\hline 917 & 2381.48 & 1319.14 & 812.67 & 253.67 \\
\hline 924 & 418.91 & 172.87 & 380.54 & 161.44 \\
\hline 931 & 1716.32 & 821.11 & 259.95 & 338.16 \\
\hline 938 & 780.61 & 614.82 & 175.53 & 158.79 \\
\hline 945 & 350.06 & 1760.38 & 316.26 & 253.82 \\
\hline 952 & 197.59 & 654.26 & 180.9 & 186.37 \\
\hline 959 & 194.91 & 81.98 & 169.76 & 125.6 \\
\hline 966 & 278.45 & 101.47 & 211.62 & 181 \\
\hline 973 & 223.35 & 428.5 & 249.58 & 156.17 \\
\hline 980 & 445.67 & 444.98 & 165.95 & 229.54 \\
\hline 987 & 144.22 & 512.62 & 150.19 & 160.4 \\
\hline 994 & 669.31 & 305.9 & 145.09 & 123.01 \\
\hline 1001 & 217.04 & 40.27 & 124.82 & 153.15 \\
\hline 1008 & 102.39 & 118.99 & 105.28 & 116.03 \\
\hline 1015 & 111.77 & 115.13 & 127.56 & 91.31 \\
\hline 1022 & 59.39 & 21.11 & 118.92 & 62.48 \\
\hline 1029 & 106.72 & 63.87 & 102.75 & 90.32 \\
\hline 1036 & 144.52 & 54.67 & 73.53 & 66.22 \\
\hline 1043 & 45.58 & 49.17 & 68.95 & 52.92 \\
\hline 1050 & 55.07 & 49.15 & 59.17 & 55.72 \\
\hline 1057 & 24.19 & 8.24 & 59.96 & 52.19 \\
\hline
\end{tabular}


Table 8: Optimality gaps (in \%) for France considering the different settings and budgets.

\begin{tabular}{|c|c|c|c|c|c|c|c|c|c|}
\hline budget & CPLEX & heuristic & cuts & heuristic + cuts & budget & CPLEX & heuristic & cuts & heuristic + cuts \\
\hline 1414 & 100 & 100 & 100 & 100 & 1715 & 30.75 & 3.41 & 3.98 & 2.85 \\
\hline 1421 & 100 & 100 & 100 & 100 & 1722 & 36.31 & 5.28 & 2.98 & 3.46 \\
\hline 1428 & 100 & 100 & 100 & 100 & 1729 & 27.12 & 3.44 & 2.46 & 5.01 \\
\hline 1435 & 100 & 100 & 100 & 100 & 1736 & 12.94 & 2.67 & 3.14 & 2.06 \\
\hline 1442 & 100 & 100 & 100 & 100 & 1743 & 32.61 & 3.23 & 5.2 & 2.98 \\
\hline 1449 & 100 & 100 & 100 & 100 & 1750 & 2.9 & 1.95 & 2.11 & 2.49 \\
\hline 1456 & 100 & 100 & 100 & 100 & 1757 & 6.05 & 2.96 & 1.56 & 2.46 \\
\hline 1463 & 100 & 100 & 100 & 100 & 1764 & 100 & 3.54 & 1.2 & 1.67 \\
\hline 1470 & 100 & 33.6 & 100 & 21.48 & 1771 & 3.67 & 3.06 & 1.56 & 1.68 \\
\hline 1477 & 100 & 100 & 100 & 100 & 1778 & 7.36 & 2.81 & 1.59 & 2.2 \\
\hline 1484 & 100 & 27.06 & 100 & 100 & 1785 & 2.06 & 3.78 & 2.24 & 2.34 \\
\hline 1491 & 100 & 14.86 & 100 & 100 & 1792 & 3.23 & 2.92 & 1.68 & 1.36 \\
\hline 1498 & 100 & 100 & 14.25 & 26.57 & 1799 & 1.9 & 2.86 & 1.61 & 2.26 \\
\hline 1505 & 100 & 19.69 & 100 & 21.43 & 1806 & 34.05 & 1.99 & 1.78 & 2.09 \\
\hline 1512 & 100 & 22.47 & 100 & 11.18 & 1813 & 1.27 & 2.22 & 1.26 & 1.64 \\
\hline 1519 & 100 & 17.77 & 100 & 12.2 & 1820 & 2.8 & 1.96 & 1.85 & 1.71 \\
\hline 1526 & 100 & 20.99 & 100 & 9.62 & 1827 & 1.44 & 1.89 & 0.55 & 1.54 \\
\hline 1533 & 100 & 19.42 & 100 & 11.91 & 1834 & 1.85 & 1.01 & 0.51 & 1.47 \\
\hline 1540 & 100 & 15.22 & 100 & 11.4 & 1841 & 0.49 & 1.88 & 0.59 & 0.54 \\
\hline 1547 & 100 & 21.01 & 100 & 7.32 & 1848 & 1.88 & 0.96 & 0.28 & 0.88 \\
\hline 1554 & 100 & 13.28 & 100 & 11.48 & 1855 & 0.27 & 1.12 & 0.17 & 0.47 \\
\hline 1561 & 100 & 15.68 & 100 & 13.34 & 1862 & 0.29 & 0.93 & 0.37 & 0.64 \\
\hline 1568 & 37.35 & 12.09 & 100 & 8 & 1869 & 0.27 & 0.55 & 0.35 & 0.94 \\
\hline 1575 & 35.2 & 9.93 & 100 & 10.33 & 1876 & 0.22 & 0.47 & 0.25 & 0.26 \\
\hline 1582 & 100 & 7.38 & 100 & 12.03 & 1883 & 0.77 & 0.81 & 0.34 & 0.51 \\
\hline 1589 & 100 & 5.72 & 100 & 4.8 & 1890 & 0.57 & 0.48 & 0.09 & 0.72 \\
\hline 1596 & 29.46 & 9.95 & 100 & 14.12 & 1897 & 0.52 & 0.35 & 0.01 & 0.17 \\
\hline 1603 & 35.19 & 8.72 & 100 & 5.35 & 1904 & 0.83 & 0.27 & 0.01 & 0.24 \\
\hline 1610 & 31.62 & 12.94 & 100 & 5.5 & 1911 & 0.01 & 0.51 & 0.01 & 0.34 \\
\hline 1617 & 100 & 7.14 & 100 & 5.33 & 1918 & 0.05 & 0.57 & 0.01 & 0.2 \\
\hline 1624 & 32.81 & 10.58 & 100 & 6.52 & 1925 & 0.2 & 0.42 & 0.04 & 0.27 \\
\hline 1631 & 48.19 & 8.39 & 100 & 5.76 & 1932 & 0.1 & 0.87 & 0.09 & 0.2 \\
\hline 1638 & 30.99 & 7.65 & 100 & 8.07 & 1939 & 0.19 & 0.36 & 0.09 & 0.16 \\
\hline 1645 & 29.08 & 6.9 & 100 & 5.51 & 1946 & 0.06 & 0.39 & 0.03 & 0.16 \\
\hline 1652 & 27.99 & 5.98 & 9.47 & 3.24 & 1953 & 0.01 & 0.17 & 0.07 & 0.15 \\
\hline 1659 & 35.32 & 7.87 & 100 & 6.39 & 1960 & 0.04 & 0.26 & 0.02 & 0.23 \\
\hline 1666 & 27.11 & 7.51 & 100 & 5.83 & 1967 & 0.01 & 0.11 & 0.01 & 0.25 \\
\hline 1673 & 32.55 & 5.13 & 5.26 & 4.09 & 1974 & 0.01 & 0.13 & 0.01 & 0.17 \\
\hline 1680 & 40.16 & 7.53 & 7.4 & 4.56 & 1981 & 0.01 & 0.03 & 0.01 & 0.04 \\
\hline 1687 & 24.22 & 4.7 & 100 & 4.55 & 1988 & 0.01 & 0.1 & 0.01 & 0.04 \\
\hline 1694 & 36.01 & 5.62 & 3.96 & 4.81 & 1995 & 0.01 & 0.01 & 0.01 & 0.01 \\
\hline 1701 & 30.77 & 4.37 & 3.91 & 5.55 & 2002 & 0.01 & 0.01 & 0.01 & 0.01 \\
\hline 1708 & 34.47 & 3.89 & 100 & 4.73 & & & & & \\
\hline
\end{tabular}

